\title{
Total synthesis of the isoquinolinium metabolite ETM-204 of Trabectidin
}

\author{
Christian Lembacher-Fadum ${ }^{1} \cdot$ Simon Gissing ${ }^{1} \cdot$ Georg Pour $^{2} \cdot$ Rolf Breinbauer $^{1}$ (D)
}

Received: 2 August 2021 / Accepted: 27 August 2021

(c) The Author(s) 2021

\begin{abstract}
Ecteinascidin-743 (Trabectidin, Trabectedin ${ }^{\circledR}$, Yondelis ${ }^{\circledR}$ ) is a synthetically obtained pharmaceutical drug originally isolated from a marine tunicate. Trabectedin is used for the chemotherapy of soft-tissue sarcoma and ovarian cancer. The isoquinolinium metabolite ETM-204 has been found in biotransformation and degradation studies of Trabectedin. We report the first total synthesis of ETM-204 and its full spectroscopic characterization confirming the postulated structure. Central elements of the 12-step synthesis starting from 2-methyl-6-nitrophenol are a $\mathrm{Cu}$-mediated conversion of an iodoarene to a phenol, a Skattebøl-formylation, and a modified Pomeranz-Fritsch cyclization to assemble the isoquinoline ring. The $\mathrm{pH}$-dependence of its visual absorbance could be clarified.
\end{abstract}

\section{Graphic abstract}

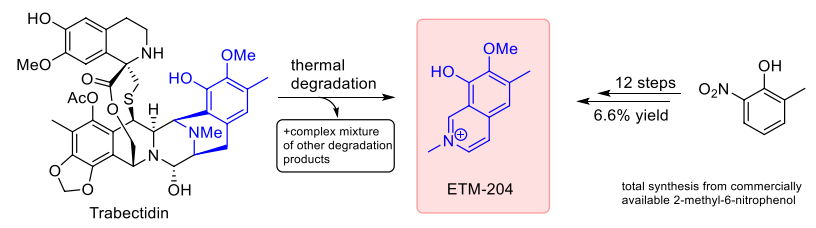

Keywords Cyclization $\cdot$ Heterocycles $\cdot$ Indicator dye $\cdot$ Medicinal chemistry $\cdot$ Ullman coupling

\section{Introduction}

Ecteicinascidin-743 (ET-743) is a marine alkaloid originally isolated from the Caribbean tunicate Ecteinascidia turbinata. ET-743 binds covalently to guanine residues in the minor groove of the DNA double helix, which triggers a series of downstream events resulting in potent antitumor activity. ET-743 is marketed as Trabectedin ${ }^{\circledR}\left(\right.$ Yondelis $\left.^{\circledR}\right)$ for the treatment of soft-tissue carcinoma and ovarian cancer. This remarkable biological activity with its unique mechanism of action together with its complex structure has made it an attractive target for total synthesis. Impressive total syntheses have been reported by Corey [1], Fukuyama [2,

Rolf Breinbauer

breinbauer@tugraz.at

1 Institute of Organic Chemistry, Graz University of Technology, Graz, Austria

2 Ever Valinject GmbH, Unterach Am Attersee, Austria
3], Danishefsky [4, 5], Zhu [6, 7], Chen [8], Williams [9], Ma [10], and several synthetic studies towards its synthesis have been undertaken [11]. Commercial production involves the semi-synthesis starting from cyanosafracin B $[12,13]$.

In the course of the clinical development of Trabectidin, studies have been undertaken, which investigated the metabolism and degradation of this complex natural product [14-17]. Several metabolites have been identified and characterized via HPLC-MS. In Scheme 1 we outline the degradation pathway, which leads to the formation of ETM204 and other metabolites proposed in the literature [14-17]. To confirm the structure of the key metabolite, which also represents a degradation product of the drug substance and product, we isolated ETM-204 and partially characterized it on the one hand and sought out to synthesize ETM-204 and confirm its structure by full spectroscopic characterization and make it accessible for biological testing and as reference standard on the other hand. 


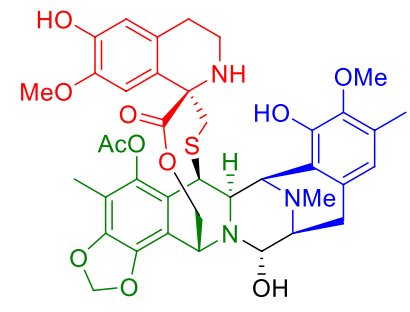

Trabectidin
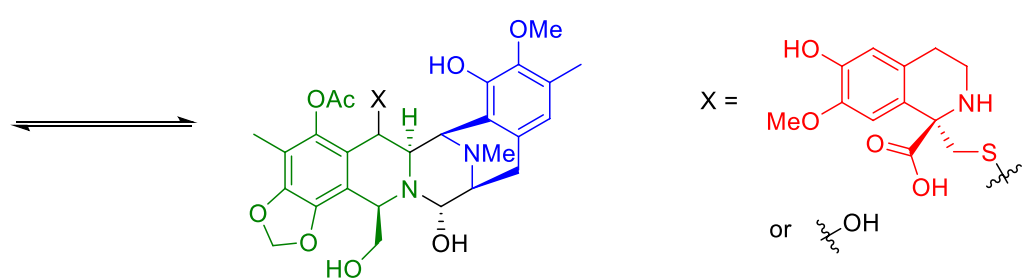

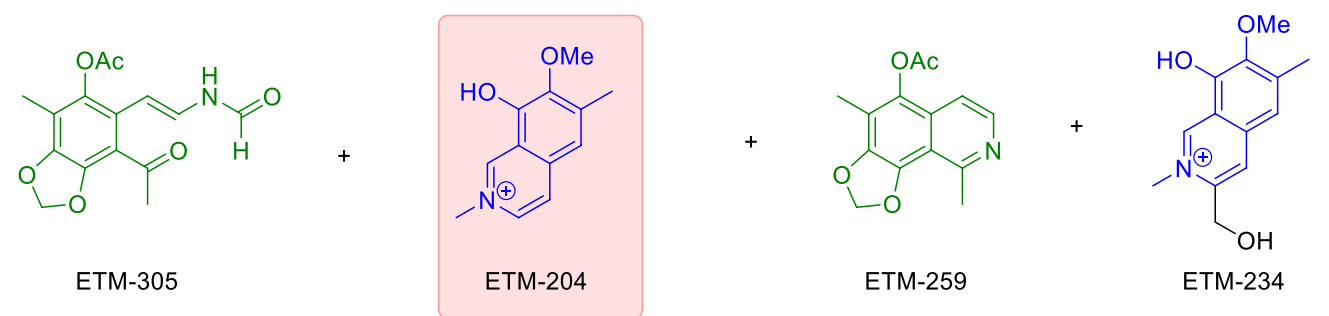

Scheme 1 Main degradation products of trabectidin proposed in the literature [14-17]

\section{Results and discussion}

\section{Isolation and characterization of degradation product ETM-204}

To verify and characterize ETM-204, one of the main degradation products, sufficient amounts of this substance needed to be isolated from a stressed Trabectedin solution. Thermal stress applied to a methanolic solution was found to be most efficient to generate high amounts of the target substance. Ideally, Trabectedin solution was incubated for $4 \mathrm{~d}$ at $60{ }^{\circ} \mathrm{C}$ yielding the degradation product in approx. 35-40\% (based on relative area by HPLC). Higher temperatures and/ or longer incubation time did not improve the yield. Using semi-preparative HPLC $2 \mathrm{mg}$ of material was purified which was subjected to NMR analysis for structure elucidation and confirmation.

Additionally HPLC-MS experiments using stressed Trabectedin solution were conducted to further characterize the degradation product. Signals from ESI positive mode at $\mathrm{m} / \mathrm{z}$ 204 as well as $\mathrm{m} / z .189$ and $\mathrm{m} / z .161$ indicate the pseudomolecular ion $(\mathrm{M}+\mathrm{H})$, the loss of $15 \mathrm{Da}(\mathrm{m} / \mathrm{z}, 204 \rightarrow 189)$ representing a methyl group and $43 \mathrm{Da}(\mathrm{m} / z, 204 \rightarrow 161)$ representing a $\mathrm{CH}_{3} \mathrm{CHN}$ fragment all shown in Fig. 1. These results are in agreement with published data from Reid [14].

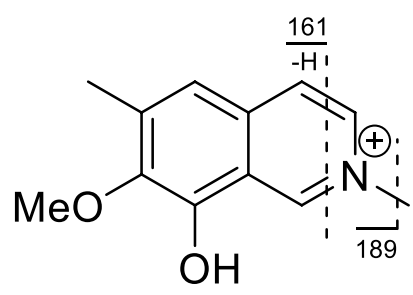

Fig. 1 Mass spectrometric fragments used for structural annotation of ETM-204

\section{Total synthesis of ETM-204}

To confirm the structure and obtain sufficient quantities for analytical and biological studies we pursued a total synthesis of ETM-204 (Scheme 2).

As starting material for our synthesis, we used commercially available 2-methyl-6-nitrophenol and converted it into nitroanisole $\mathbf{1}$ via Williamson ether synthesis using $1.5 \mathrm{eq}$ iodomethane and $2.0 \mathrm{eq}$ potassium carbonate in DMF at $23{ }^{\circ} \mathrm{C}$. This reaction delivered $\mathbf{1}$ in excellent $99 \%$ yield after aqueous workup and sufficient purity, which allowed to use the crude product of $\mathbf{1}$ directly in a reduction of the nitro group with 5.0 eq iron powder in an acetic acid/water mixture to produce $o$-anisidine 2 . Although the 


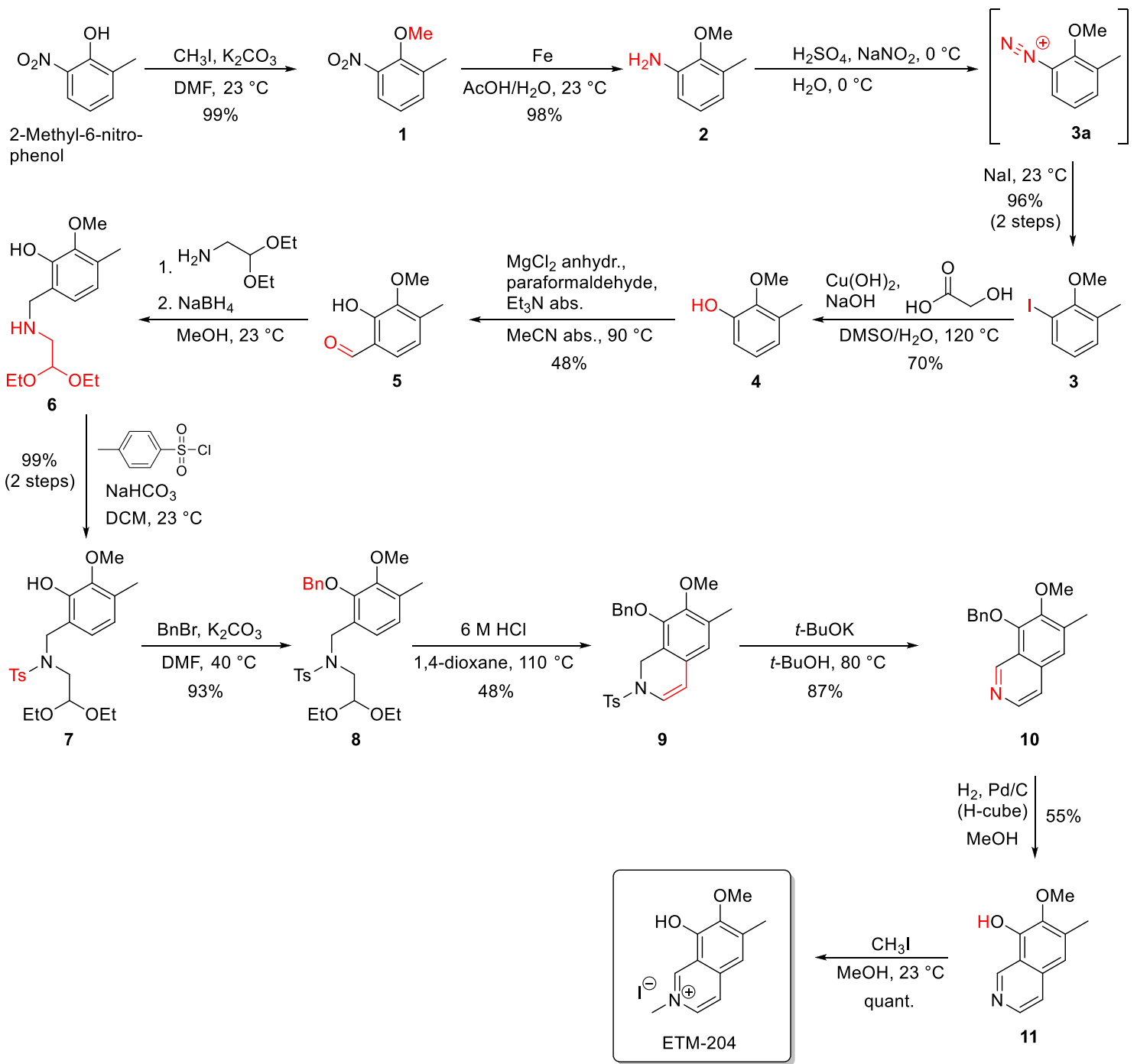

Scheme 2 Synthetic route towards Trabectedin degradation product ETM-204

phase separation during aqueous workup took some time because of formation of an emulsion, the reaction provided 98\% of the desired compound 2 as a red-brown oil, which was already pure according to NMR and used without further treatment. A colorless product sample was obtained via distillation with a Kugelrohr apparatus.

Next, we planned to convert the amino group of compound 2 into phenol-OH by first forming the corresponding aryl diazonium salt $\mathbf{3 a}$ and subsequent replacement by a hydroxy group via heating up the acidic aqueous solution to obtain 3-methylguajacol (4) directly. While the diazotation worked reliably, we failed to convert the diazonium species 3a to compound 4, as it either did not react at all, or decomposed after longer reaction times. Therefore, we decided to use iodo compound $\mathbf{3}$ as an intermediate, which was formed in $96 \%$ yield after addition of 2.1 eq sodium iodide solution to the diazonium intermediate $\mathbf{3 a}$ and stirring at $23{ }^{\circ} \mathrm{C}$ overnight. The conversion of iodoarene $\mathbf{3}$ into phenol $\mathbf{4}$ was planned using copper salts as catalyst and water as a nucleophile [18]. The best yield (70\%) was achieved using a procedure of Xiao et al., with $\mathrm{Cu}(\mathrm{OH})_{2}$ in combination with glycolic acid as ligand [19].

The next step involved the ortho-formylation of phenol 4. First attempts using $\mathrm{SnCl}_{4}$ /paraformaldehyde [20] or hexamethylenetetramine [21, 22] failed for this substrate. Next, we tried a procedure of Hofsløkken and Skattebøl, who described a convenient method for the ortho-formylation of phenols using $\mathrm{MgCl}_{2} / \mathrm{Et}_{3} \mathrm{~N}$ and paraformaldehyde in dry $\mathrm{MeCN}$ [23]. First test reactions were encouraging, but the yield was very low. We finally achieved the best results with fresh batches of $\mathrm{MgCl}_{2}$ and paraformaldehyde, which have been dried for $90 \mathrm{~min}$ in oil-pump vacuum before they were used, and isolated $\mathbf{5}$ in moderate $48 \%$ yield. 
A

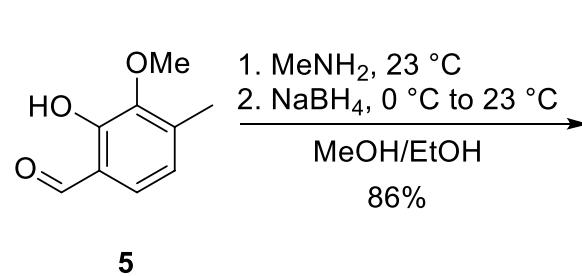

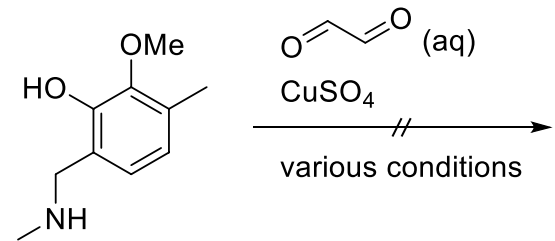

$6 a$<smiles>COc1c(C)cc2cc[n+](C)cc2c1O</smiles>

ETM-204

B<smiles>COc1c(C)ccc(C=O)c1O</smiles><smiles>CCOC(CN)OCC</smiles><smiles>CCOC(C/N=C\c1ccc(C)c(OC)c1O)OCC</smiles>

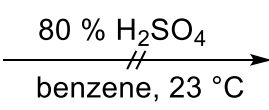<smiles>COc1c(C)cc2ccncc2c1O</smiles>
11

Scheme 3 Unsuccessful cyclization strategies A via Pictet-Spengler reaction of $\mathbf{6 a}$ and $\mathbf{B}$ via Pomeranz-Fritsch reaction directly from intermediate $\mathbf{6 b}$

Our initial strategy towards the $N$-methylated isoquinoline scaffold was based on the synthesis of compound $\mathbf{6 a}$ via reductive amination, followed by a Pictet-Spengler reaction with glyoxal (Scheme 3). As previous publications indicated that suitable reaction conditions could lead directly to the oxidized form of the heterocycle [24], we tried several conditions (variation of the temperature, changing the amount of glyoxal solution, using formic acid, acetic acid or 1,4-dioxane/HCl). Unfortunately, despite these efforts we could not detect any cyclized product. As an alternative cyclization method, we attempted a Pomeranz-Fritsch reaction [25] by condensing aldehyde 5 with aminoacetaldehyde dimethyl acetal to Schiff base $\mathbf{6 b}$ (Scheme 3). Unfortunately, it was not possible to cyclize $\mathbf{6 b}$ directly under acidic conditions as described by Woodward and Doering for a related, but less complex substrate [26]. Therefore, we followed a modified cyclization strategy of Birch and coworkers [27] by first reducing imine $\mathbf{6 b}$ with sodium borohydride to amine 6, which was directly N-tosylated without further purification to obtain 7 in excellent $99 \%$ yield over 2 steps. As many by-products were detected in the cyclization attempts of 7 we speculated that we could improve the selectivity of the reaction, by protecting the phenol-OH as a benzyl ether (Scheme 2). Indeed cyclization of 8 in 1,4-dioxane/6 M aqueous $\mathrm{HCl}$ at $110{ }^{\circ} \mathrm{C}$ led to cyclized product 9 in $48 \%$ yield. The expected elimination of the tosyl group did not proceed under acidic conditions, probably because of the electron donating effect of the benzyloxy substituent in position 8 , which renders the proton in position 1 less acidic [27]. However, this could be easily overcome by treating 9 with potassium tert-butoxide in tert-butanol to form isoquinoline $\mathbf{1 0}$ in $87 \%$ yield (Scheme 2).

After deprotection of $\mathbf{1 0}$ via catalytic hydrogenation, using an $\mathrm{H}$-cube ${ }^{\circledR}$ continuous-flow hydrogenation reactor with a $10 \% \mathrm{Pd}$ on charcoal cartridge, 11 was obtained in moderate $55 \%$ yield after flash column chromatography, where also unreacted starting material $\mathbf{1 0}$ was collected and subjected to a second hydrogenation cycle via H-cube ${ }^{\circledR}$.

With purified isoquinoline $\mathbf{1 1}$ in hand, a few test reactions were performed to find the best conditions for the methylation of the nitrogen atom. We had concerns that the phenolic $\mathrm{OH}$ could be methylated as well, which would complicate the purification of the final product. This issue could be avoided by exclusion of any addition of a base. Even with a large excess of iodomethane exclusive N-methylation was observed. Pure target compound ETM-204 was obtained in quantitative yield as an iodide salt by evaporation of the volatiles and subsequent drying in oil-pump vacuum (Scheme 2).

The final product was orange-colored as a solid and solutions were yellow (dissolved in methanol or chloroform), which was in contrast to the deep red color of the material isolated from degradation studies described above. Furthermore, all aromatic protons in our ${ }^{1} \mathrm{H}$ spectrum were 

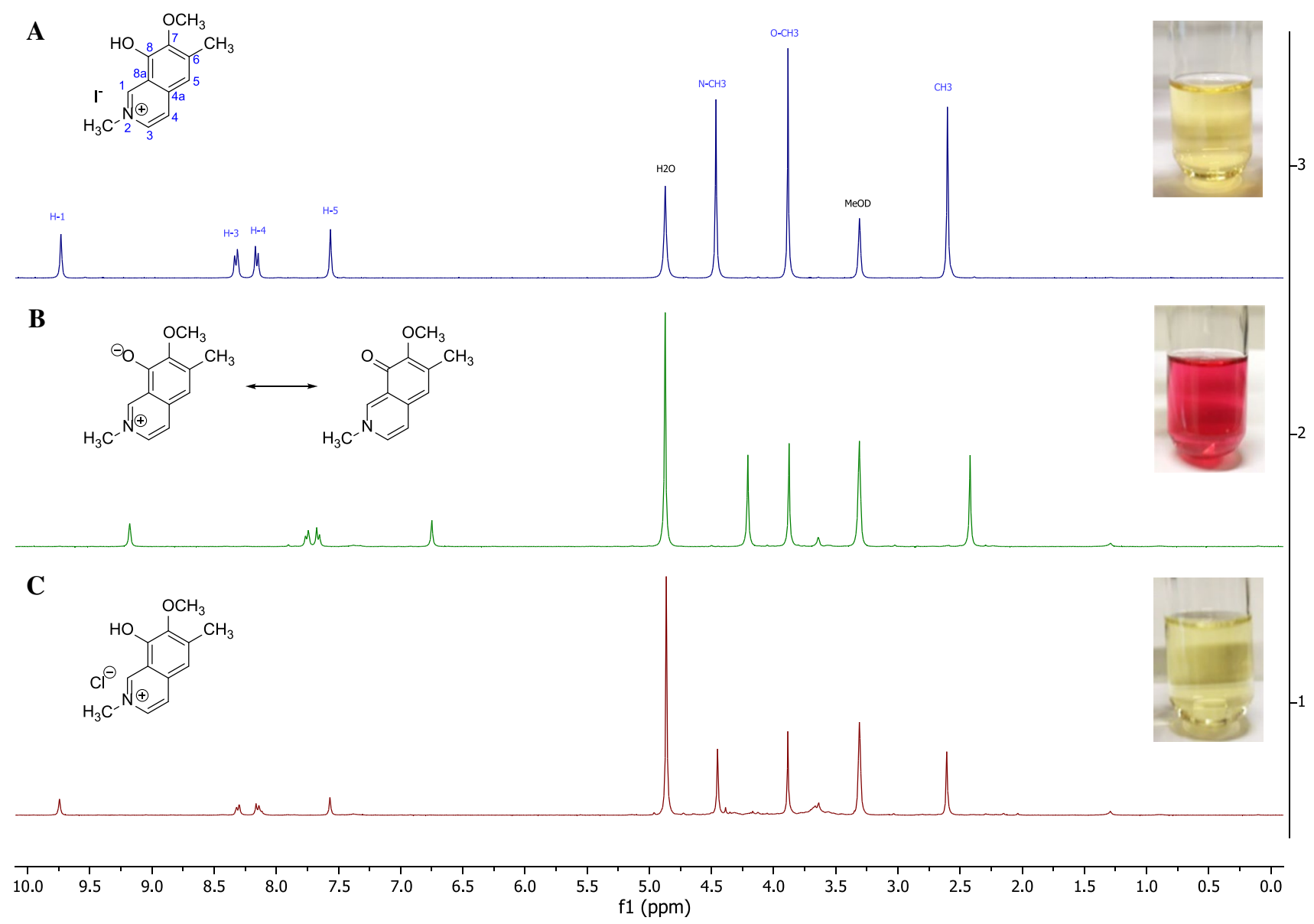

Fig. 2 pH-dependent behavior of ETM-204 in methanolic solution and the corresponding ${ }^{1} \mathrm{H}$ NMR spectra. HPLC traces of the samples are provided in the supporting information

significantly shifted downfield compared to the ${ }^{1} \mathrm{H}$ spectrum of Reid et al. [14], while the HPLC retention times of synthetic and isolated products were identical. To understand this different appearance, we performed experiments to study the acid/base behavior of ETM-204. Figure 2A shows the ${ }^{1} \mathrm{H}$ NMR spectrum of the synthesized compound (with iodide as counter ion) and its solution in methanol. Next, we dissolved a small amount of our compound in $1 \mathrm{M} \mathrm{NaOH}$ (orange solution), and extracted it with chloroform, where the organic phase immediately turned red. After phase separation, drying of the organic phase over $\mathrm{Na}_{2} \mathrm{SO}_{4}$ and filtration, the volatiles were removed under reduced pressure and a dark red solid was obtained, which was dissolved in methanol- $d_{4}$ and a ${ }^{1} \mathrm{H}$ NMR spectrum was recorded (Fig. 2B). The proton shifts of this betaine compound were in full alignment with the values previously described in literature [14]. After addition of a droplet of $4 \mathrm{M} \mathrm{HCl}$ in 1,4-dioxane to the red solution, the color immediately turned yellow again (Fig. 2C). After evaporation of the volatiles, a third ${ }^{1} \mathrm{H}$ spectrum was recorded, which showed the same shifts as the initial spectrum and reflects the phenolic form of ETM-204.

\section{Conclusion}

In summary, we have successfully synthesized the central Trabectedin metabolite ETM-204 in a 12-step synthesis, assigned its structure via NMR spectroscopy and confirmed it with authentic material resulting from degradation studies. The synthesis started from commercially available 2-methyl-6-nitrophenol, which was converted into 2-methoxy-3-methylphenol (4) in four steps. ortho-Formylation with paraformaldehyde and $\mathrm{MgCl}_{2} / \mathrm{Et}_{3} \mathrm{~N}$ provided 2-hydroxy-3-methoxy-4-methylbenzalydehyde (5), which was further subjected to a reductive amination, followed by an N-tosylation. Key step of the synthesis was the cyclization to 1,2-dihydroisoquinoline derivative $\mathbf{9}$, which only succeeded after protection of the phenol-OH with a benzyl group. After aromatization and deprotection, isoquinoline 11 was finally $\mathrm{N}$-methylated with an excess of iodomethane to yield ETM-204 as iodide. We could show that ETM-204 shows a pH-dependent behavior and can either exist as a cationic species in acidic $\mathrm{pH}$ or as a betaine under basic $\mathrm{pH}$, which has to be considered when investigating its properties. 


\section{Experimental}

Reactions were carried out under air, unless indicated otherwise. For inert reactions, standard Schlenk techniques under an inert atmosphere of $\mathrm{N}_{2}$ or $\mathrm{Ar}$ and anhydrous solvents were used. The described nuclear resonance spectra were acquired with the following instruments: Bruker AVANCE III with autosampler: $300.36 \mathrm{MHz}{ }^{1} \mathrm{H}$ NMR, $75.53 \mathrm{MHz}{ }^{13} \mathrm{C}$ NMR; chemical shifts $\delta[\mathrm{ppm}]$ are referenced to residual protonated solvent signals as internal standard: $\mathrm{CDCl}_{3}: \delta=7.26 \mathrm{ppm}$ $\left({ }^{1} \mathrm{H}\right), 77.16 \mathrm{ppm}\left({ }^{13} \mathrm{C}\right)$; methanol- $d_{4}: \delta=3.31 \mathrm{ppm}\left({ }^{1} \mathrm{H}\right)$, $49.00 \mathrm{ppm}\left({ }^{13} \mathrm{C}\right)$. Signal multiplicities are abbreviated as bs (broad singlet), d (doublet), dd (doublet of doublet), dt (doublet of triplet), $m$ (multiplet), s (singlet), $t$ (triplet), and q (quadruplet). The deuterated solvent, the chemical shifts $\delta$ in ppm (parts per million), and the coupling constants $J$ in Hertz $(\mathrm{Hz})$ are given. Deuterated solvents for nuclear resonance spectroscopy were purchased from Euriso-top ${ }^{\circledR}$. Analytical thin layer chromatography (TLC) was performed on Merck silica gel $60, \mathrm{~F}_{254}$ plates and spots were visualized by UV-light ( $\lambda=254$ and/or $366 \mathrm{~nm}$ ), or by treatment with cerium ammonium molybdate solution (CAM: $5.0 \mathrm{~g}$ $\mathrm{Ce}(\mathrm{IV}) \mathrm{SO}_{4}, 50 \mathrm{~g}\left(\mathrm{NH}_{4}\right)_{2} \mathrm{MoO}_{4}, 50 \mathrm{~cm}^{3}$ concentrated $\mathrm{H}_{2} \mathrm{SO}_{4}$ in $450 \mathrm{~cm}^{3}$ water) or $\mathrm{KMnO}_{4}$ solution $\left(3.0 \mathrm{~g} \mathrm{KMnO}_{4}\right.$ and $20.0 \mathrm{~g} \mathrm{~K}_{2} \mathrm{CO}_{3}$ dissolved in $300 \mathrm{~cm}^{3} \mathrm{H}_{2} \mathrm{O}$ and $5 \mathrm{~cm}^{3}$ of $5 \%$ aqueous $\mathrm{NaOH}$ ). Flash column chromatography was performed using silica gel 60 from Macherey-Nagel (40-63 $\mu \mathrm{m}$ particle size) at an air pressure of $\sim 1.5$ bar. Analytical HPLC measurements were performed on an Agilent Technologies 1200 Series HPLC system with 1260 HiP Degasser G4225A, binary pump SL G1312, autosampler HiP-ALS SL G1367C, thermostated column compartment TCC SL G1316B, multiple wavelength detector G1365C MWD SL with deuterium lamp $(\lambda=190-400 \mathrm{~nm})$ and subsequent connected mass detector (Agilent Technologies 6120 Quadrupole LC/MS) with an electrospray ionization (ESI) source. The components were separated on a RP Agilent Poroshell 120 EC-C18 column $(3.0 \times 100 \mathrm{~mm}, 2.7 \mu \mathrm{m}$ particles $)$ with a Merck LiChroCART $^{\circledR}$ 4-4 pre-column. Signals were detected at 210 or $254 \mathrm{~nm}$. As mobile phase acetonitrile (VWR HiPerSolv, HPLC-MS grade) and water (Barnstead NANOpure ${ }^{\circledR}$, ultrapure water system) with $0.05 \%$ trifluoroacetic acid (TFA) was used. The following standard method was used: Column oven: $40{ }^{\circ} \mathrm{C}$, flow rate $0.7 \mathrm{~cm}^{3} / \mathrm{min} ; 0-0.5 \mathrm{~min}$ $\mathrm{CH}_{3} \mathrm{CN} / 0.05 \%$ TFA $=2: 98(\mathrm{v} / \mathrm{v}), 0.5-10.0$ min linear increase to $\mathrm{CH}_{3} \mathrm{CN} / 0.05 \%$ TFA $=100: 0(\mathrm{v} / \mathrm{v}), 10.0-12.0 \mathrm{~min}$ hold $\mathrm{CH}_{3} \mathrm{CN} / 0.05 \% \mathrm{TFA}=100: 0(\mathrm{v} / \mathrm{v}), 12.5-13.0 \mathrm{~min}$ return to initial conditions. High-resolution mass spectrometry (HRMS): all HRMS measurements were done at Vienna BioCenter, using the following procedure: sample spectra were acquired by data-dependent high-resolution tandem mass spectrometry on a QExactive Focus (Thermo Fisher Scientific, Germany). The electrospray ionization potential was set to +3.5 or $-3.0 \mathrm{kV}$, the sheath gas flow was set to 20 , and an auxiliary gas flow of 5 was used. Samples were diluted with an appropriate solvent (methanol or chloroform) and $1 \mathrm{~mm}^{3}$ was injected on a SeQuant ${ }^{\circledR}$ ZIC $^{\circledR}$-pHILIC HPLC column (Merck, $100 \times 2.1 \mathrm{~mm} ; 5 \mu \mathrm{m} ; 100 \AA$; peek coated; equipped with a guard column). The separation solvent (A: ACN, B: $25 \mathrm{mM} \mathrm{ABC}$ ) was delivered through an Ultimate 3000 HPLC system (Thermo Fisher Scientific, Germany) with a flow rate of $100 \mathrm{~mm}^{3} \mathrm{~min}^{-1}$ and appropriate gradients were used for proper sample elution. Acetonitrile (ACN) HiPerSolv CHROMANORM ${ }^{\circledR}$ for HPLC-Supergradient was obtained from VWR Chemicals, methanol Optima ${ }^{\circledR}$ LC/MS Grade from Fisher Chemicals, ammonium hydrogen carbonate for LC-MS LiChropur were purchased from Merck, chloroform Plus for HPLC from Sigma-Aldrich, and $\mathrm{H}_{2} \mathrm{O}$ was obtained from a Milli- $\mathrm{Q}^{\circledR}$ Advantage A10 water purification system (Merck). Melting points were measured on a MEL-TEMP $^{\circledR}$ apparatus with integrated microscopical support from Electrothermal in open capillary tubes. Reported values are uncorrected. Hydrogenation experiments were performed using the H-Cube ${ }^{\circledR}$ continuous-flow hydrogenation unit (HC-2.SS) from ThalesNano Inc. running with a Knauer Smartline pump 100 and equipped with a $10 \mathrm{~cm}^{3}$ ceramic pump head. As hydrogenation catalyst $10 \% \mathrm{Pd} / \mathrm{C}$ catalyst cartridges were used (ThalesNano Inc., THS01111, $10 \% \mathrm{Pd} / \mathrm{C} \mathrm{CatCart}^{\mathrm{TM}}$ ). Chemicals were purchased from the companies ABCR, ACROS Organics, Alfa Aesar, Brenntag, Fisher Scientific, Fluka, Merck, Roth, Sigma Aldrich or VWR and were used without further purification, unless otherwise stated. All solvents were purchased from the abovementioned companies and were used without further purification unless otherwise stated. For reactions where moisture was excluded, absolute solvents were used. For that purpose, the purchased solvents were dried using the following methods and stored in brown $1 \mathrm{dm}^{3}$ Schlenk bottles under argon and over activated molecular sieves. For analytical applications solvents with analytical grade were purchased. Absolute $\mathrm{DMF}, \mathrm{MeCN}$, and $\mathrm{MeOH}$ were purchased in anhydrous quality and stored over activated molecular sieves $(3 \AA$ : $\mathrm{MeCN}, \mathrm{MeOH} ; 4$ A: DMF) under argon. Dichloromethane: $\mathrm{CH}_{2} \mathrm{Cl}_{2}$ (stabilized with EtOH) was first heated under reflux over $\mathrm{P}_{4} \mathrm{O}_{10}$ for $5 \mathrm{~h}$, then heated under reflux over $\mathrm{CaH}_{2}$ for $5 \mathrm{~h}$ and distilled under argon atmosphere into a brown $1 \mathrm{dm}^{3}$ Schlenk bottle containing activated $4 \AA$ molecular sieves. Absolute $\mathrm{Et}_{3} \mathrm{~N}$ was obtained by refluxing over $\mathrm{CaH}_{2}$ and distilling into a $1 \mathrm{dm}^{3}$ Schlenk bottle containing activated $4 \AA$ molecular sieves. 1,4-Dioxane was distilled and stored over $\mathrm{KOH}$.

\section{Isolation of ETM-204 by degradation of Trabectedin}

$20 \mathrm{mg}$ Trabectedin were placed into a $10 \mathrm{~cm}^{3}$ glass vial, dissolved in $8 \mathrm{~cm}^{3} \mathrm{MeOH}$, closed and put into an oven 
for $4 \mathrm{~d}$ at $60{ }^{\circ} \mathrm{C}$. Afterwards the reaction was stopped by placing the solution into a fridge. Semi-preparative chromatography of stressed solutions was conducted on an Agilent 1260 Infinity II instrument with quaternary pump G7111B, sampler G7129A, thermostatic column compartment G7116B and DAD G7115A using a Zorbax SB-Phenyl, $250 \times 9.6 \mathrm{~mm}$ with $5 \mu \mathrm{m}$ particles column. Respective fractions were collected automatically during several runs using a G1364 Agilent fraction collector. Mobile phase A was an aqueous solution with $16 \mathrm{mM}$ ammonium formate (Fluka, LC-grade) and $26 \mathrm{mM}$ formic acid (Merck, ACS Reag Ph Eur.). As mobile phase B acetonitrile was used (VWR, LC-grade). First step of the run consisted of $10 \mathrm{~min}$ isocratic phase at a composition of $82 \% \mathrm{~A}$ and $18 \% \mathrm{~B}$ and a flow rate of $4.5 \mathrm{~cm}^{3} / \mathrm{min}$. After that the column was washed by changing to $90 \%$ mobile phase B for $11 \mathrm{~min}$ followed by an equilibration step of $5 \mathrm{~min}$. Collected fractions were combined and concentrated using evaporator GeneVac EZ-2 (Bartelt). Remaining residues were resuspended in $1.5 \mathrm{~cm}^{3}$ dichloromethane (VWR, GCgrade). The supernatant was carefully removed from the precipitate and put into a fresh HPLC-vial. Again, material was evaporated to complete dryness to yield $2 \mathrm{mg}$ and stored at $2-8{ }^{\circ} \mathrm{C}$ until NMR analysis. HPLC-MS analysis of solutions from degradation study was performed on an Agilent 1290 Infinity instrument with binary pump G4220A, sampler GG4226A, thermostatic column compartment G1316C and DAD G4212A coupled with a Sciex QTRAP 4500 operating in positive electron spray mode $($ ESI +$)$. Components were separated on a Zorbax SBPhenyl, $150 \times 4.6 \mathrm{~mm}$ with $1.8 \mu \mathrm{m}$ particles column. The same mobile phases were used as described above but in MS-grade quality. At a flow rate of $0.8 \mathrm{~cm}^{3} / \mathrm{min}$ initial conditions were $15 \%$ mobile phase B increasing to $27 \%$ after $45 \mathrm{~min}$. A second steeper gradient was implemented until $75 \mathrm{~min}$ up to $65 \% \mathrm{~B}$ followed by an equilibration step for $12 \mathrm{~min}$ at initial conditions.

2-Methoxy-1-methyl-3-nitrobenzene (1) A dried and argon-flushed $100 \mathrm{~cm}^{3}$ Schlenk-flask with magnetic stirring bar was charged with $5.66 \mathrm{~g}$ 2-methyl-6-nitrophenol (37.0 mmol, $1.0 \mathrm{eq}$ ), which were then dissolved in $70 \mathrm{~cm}^{3}$ abs. DMF forming a yellow solution. Then $10.2 \mathrm{~g} \mathrm{~K}_{2} \mathrm{CO}_{3}$ (73.9 mmol, $2.0 \mathrm{eq}$ ) and $3.50 \mathrm{~cm}^{3}$ iodomethane $(55.4 \mathrm{mmol}$, $1.5 \mathrm{eq})$ were added resulting in a red suspension, which was stirred for $60 \mathrm{~h}$ at $23{ }^{\circ} \mathrm{C}$. The suspension, which had turned orange, was diluted with $300 \mathrm{~cm}^{3}$ EtOAc, poured into a $1000 \mathrm{~cm}^{3}$ separation funnel and washed with halfsatured $\mathrm{NaCl}$ solution $\left(5 \times 300 \mathrm{~cm}^{3}\right)$ and brine $(1 \times 200$ $\mathrm{cm}^{3}$ ). The resulting yellow organic layer was dried over $\mathrm{Na}_{2} \mathrm{SO}_{4}$, filtered and concentrated on a rotary evaporator furnishing $6.13 \mathrm{~g} 1$ (36.7 mmol, 99\%) as a red oil. $R_{f}=0.48$ $\left(\right.$ cyclohexane/EtOAc $\left.=10: 1(\mathrm{v} / \mathrm{v}) ; \mathrm{UV} / \mathrm{KMnO}_{4}\right) ;{ }^{1} \mathrm{H} \mathrm{NMR}$ $\left(300 \mathrm{MHz}, \mathrm{CDCl}_{3}\right): \delta=7.63(\mathrm{~d}, J=7.4 \mathrm{~Hz}, 1 \mathrm{H}, \mathrm{Ar}-\mathrm{H}), 7.41$ (d, $J=7.2 \mathrm{~Hz}, 1 \mathrm{H}, \mathrm{Ar}-\mathrm{H}), 7.10(\mathrm{t}, J=7.9 \mathrm{~Hz}, 1 \mathrm{H}, \mathrm{Ar}-\mathrm{H})$, $3.90\left(\mathrm{~s}, 3 \mathrm{H}, \mathrm{OCH}_{3}\right), 2.37\left(\mathrm{~s}, 3 \mathrm{H}, \mathrm{CH}_{3}\right) \mathrm{ppm} ;{ }^{13} \mathrm{C} \mathrm{NMR}$ $\left(75.5 \mathrm{MHz}, \mathrm{CDCl}_{3}\right): \delta=151.8\left(\mathrm{C}_{\mathrm{Ar}}-\mathrm{OCH}_{3}\right), 144.5\left(\mathrm{C}_{\mathrm{Ar}}-\right.$ $\left.\mathrm{NO}_{2}\right), 135.7\left(\mathrm{C}_{\mathrm{Ar}}-\mathrm{H}\right), 134.6\left(\underline{\mathrm{C}}_{\mathrm{Ar}}-\mathrm{CH}_{3}\right), 123.9\left(\mathrm{C}_{\mathrm{Ar}}-\mathrm{H}\right)$, $123.0\left(\mathrm{C}_{\mathrm{Ar}}-\mathrm{H}\right), 62.0\left(\mathrm{OCH}_{3}\right), 16.2\left(\mathrm{CH}_{3}\right) \mathrm{ppm}$.

2-Methoxy-3-methylaniline (2) In an inert $500 \mathrm{~cm}^{3}$ round-bottom flask equipped with a Schlenk adapter and a magnetic stirring bar $6.00 \mathrm{~g}$ 2-methoxy-1-methyl-3-nitrobenzene $(1,35.9 \mathrm{mmol}, 1.0 \mathrm{eq})$ were dissolved in 180 $\mathrm{cm}^{3}$ glacial acetic acid and $18 \mathrm{~cm}^{3}$ dist. $\mathrm{H}_{2} \mathrm{O} .10 .0 \mathrm{~g}$ Fe powder $(180 \mathrm{mmol}, 5.0$ eq) were added, the Schlenk adapter connected to a bubbler and the reaction mixture stirred for $17 \mathrm{~h}$ at $23{ }^{\circ} \mathrm{C}$. The resulting grey-blue suspension was poured into a $2 \mathrm{dm}^{3}$ Erlenmeyer flask and neutralized with $400 \mathrm{~cm}^{3}$ satd. $\mathrm{K}_{2} \mathrm{CO}_{3}$-solution under ice cooling. After further dilution with $500 \mathrm{~cm}^{3}$ dist. $\mathrm{H}_{2} \mathrm{O}$ the reaction mixture was transferred into a $2 \mathrm{dm}^{3}$ separation funnel. The reaction mixture was extracted with $350 \mathrm{~cm}^{3} \mathrm{CH}_{2} \mathrm{Cl}_{2}$ upon which a third intermediate phase was formed, which was collected separately. The aqueous phase was again extracted with 350 $\mathrm{cm}^{3} \mathrm{CH}_{2} \mathrm{Cl}_{2}$ and the intermediate phase was extracted with $150 \mathrm{~cm}^{3} \mathrm{CH}_{2} \mathrm{Cl}_{2}$. The combined organic phases were dried over $\mathrm{Na}_{2} \mathrm{SO}_{4}$, filtered and the solvent removed on a rotatory evaporator (at water bath temperature $\leq 30{ }^{\circ} \mathrm{C}$ ). The resulting $4.84 \mathrm{~g}$ red-brown oil of $2(35.3 \mathrm{mmol}, 98 \%)$ was used in the next step without further purification. $R_{f}=0.16$ (cyclohexane/EtOAc $\left.=10: 1(\mathrm{v} / \mathrm{v}) ; \mathrm{UV} / \mathrm{KMnO}_{4}\right) ;{ }^{1} \mathrm{H}$ NMR $\left(300 \mathrm{MHz}, \mathrm{CDCl}_{3}\right): \delta=6.83(\mathrm{t}, J=7.7 \mathrm{~Hz}, 1 \mathrm{H}, \mathrm{Ar}-\mathrm{H}), 6.59$ ("t", $J=7.12 \mathrm{H}, \mathrm{Ar}-\mathrm{H}), 3.76$ (s, $3 \mathrm{H}, \mathrm{OCH}_{3}$, overlapping with bs, $\left.2 \mathrm{H}, \mathrm{NH}_{2}\right), 2.28\left(\mathrm{~s}, 3 \mathrm{H}, \mathrm{CH}_{3}\right) \mathrm{ppm} ;{ }^{13} \mathrm{C} \mathrm{NMR}(75.5 \mathrm{MHz}$, $\left.\mathrm{CDCl}_{3}\right): \delta=145.9\left(\underline{\mathrm{C}}_{\mathrm{Ar}}-\mathrm{OCH}_{3}\right), 139.9\left(\underline{\mathrm{C}}_{\mathrm{Ar}}-\mathrm{NH}_{2}\right), 131.2$ $\left.\left(\underline{\mathrm{C}}_{\mathrm{Ar}}-\mathrm{CH}_{3}\right), 124.5 \mathrm{C}_{\mathrm{Ar}}-\mathrm{H}\right), 120.9\left(\mathrm{C}_{\mathrm{Ar}}-\mathrm{H}\right), 113.9\left(\mathrm{C}_{\mathrm{Ar}}-\mathrm{H}\right)$, $59.8\left(\mathrm{OCH}_{3}\right), 15.9\left(\mathrm{CH}_{3}\right) \mathrm{ppm}$.

1-lodo-2-methoxy-3-methylbenzene (3) In an inert $500 \mathrm{~cm}^{3}$ round-bottom flask equipped with a Schlenk adapter and a magnetic stirring bar $4.56 \mathrm{~cm}^{3}$ 2-methoxy3-methylanilin (2, $34.6 \mathrm{mmol}, 1.0 \mathrm{eq})$ were dissolved 125 $\mathrm{cm}^{3} \mathrm{H}_{2} \mathrm{SO}_{4}\left(10 \%\right.$ in $\left.\mathrm{H}_{2} \mathrm{O}, 134.9 \mathrm{mmol}, 3.9 \mathrm{eq}\right)$ and cooled in an ice bath to $0{ }^{\circ} \mathrm{C}$. With a syringe pump a solution of $5.01 \mathrm{~g} \mathrm{NaNO}_{2}(72.6 \mathrm{mmol}, 2.1 \mathrm{eq})$ in $35 \mathrm{~cm}^{3}$ dist. $\mathrm{H}_{2} \mathrm{O}$ were added dropwise with a rate of $40 \mathrm{~cm}^{3} / \mathrm{h}$. After completed addition the resulting slightly brownish solution was stirred for another $45 \mathrm{~min}$ at $0{ }^{\circ} \mathrm{C}$. Then, a solution of $10.9 \mathrm{~g} \mathrm{NaI}$ (72.6 mmol, $2.1 \mathrm{eq}$ ) in $35 \mathrm{~cm}^{3}$ dist. $\mathrm{H}_{2} \mathrm{O}$ was added with a syringe pump at a flowrate of $4 \mathrm{~cm}^{3} / \mathrm{min}$, upon which immediately the solution turned dark-brown and foam formation was observed. After removal of the ice bath the septum of the flask was replaced with a bubbler and the reaction mixture was stirred for $19 \mathrm{~h}$ at $23{ }^{\circ} \mathrm{C}$. The resulting two-phase solution (dark brown aqueous phase and black oil) were 
transferred into a $1000 \mathrm{~cm}^{3}$ separation funnel and extracted with $\mathrm{CH}_{2} \mathrm{Cl}_{2}\left(2 \times 200 \mathrm{~cm}^{3}\right)$. The combined organic phases were then washed with $1.8 \mathrm{M} \mathrm{Na}_{2} \mathrm{~S}_{2} \mathrm{O}_{4}$ solution $(3 \times 200$ $\left.\mathrm{cm}^{3}\right)$, dist. $\mathrm{H}_{2} \mathrm{O}\left(1 \times 200 \mathrm{~cm}^{3}\right)$, and brine $\left(1 \times 200 \mathrm{~cm}^{3}\right)$, dried over $\mathrm{Na}_{2} \mathrm{SO}_{4}$, filtrated and concentrated on a rotary evaporator resulting in $8.28 \mathrm{~g} 3(33.4 \mathrm{mmol}, 96 \%)$ and dark brown oil, which was used in the next step without further purification. ${ }^{1} \mathrm{H}$ NMR $\left(300 \mathrm{MHz}, \mathrm{CDCl}_{3}\right): \delta=7.61$ (d, $J=7.8 \mathrm{~Hz}, 1 \mathrm{H}, \mathrm{Ar}-\mathrm{H}), 7.14(\mathrm{~d}, J=7.4 \mathrm{~Hz}, 1 \mathrm{H}, \mathrm{Ar}-\mathrm{H})$, $6.75(\mathrm{t}, J=7.7 \mathrm{~Hz}, 1 \mathrm{H}, \mathrm{Ar}-\mathrm{H}), 3.78\left(\mathrm{~s}, 3 \mathrm{H}, \mathrm{OCH}_{3}\right), 2.35(\mathrm{~s}$, $\left.3 \mathrm{H}, \mathrm{CH}_{3}\right) \mathrm{ppm} ;{ }^{13} \mathrm{C}$ NMR $\left(75.5 \mathrm{MHz}, \mathrm{CDCl}_{3}\right): \delta=158.2$ $\left(\underline{\mathrm{C}}_{\mathrm{Ar}}-\mathrm{OCH}_{3}\right), 137.3\left(\mathrm{C}_{\mathrm{Ar}}-\mathrm{H}\right), 132.5\left(\mathrm{~s}, 1 \mathrm{C}, \underline{\mathrm{C}}_{\mathrm{Ar}}-\mathrm{CH}_{3}\right), 131.7$ (s, $\left.1 \mathrm{C}, \mathrm{C}_{\mathrm{Ar}}-\mathrm{H}\right), 126.0\left(\mathrm{~s}, 1 \mathrm{C}, \mathrm{C}_{\mathrm{Ar}}-\mathrm{H}\right), 92.1\left(\mathrm{~s}, 1 \mathrm{C}, \underline{\mathrm{C}}_{\mathrm{Ar}}-\mathrm{I}\right)$, $60.3\left(\mathrm{~s}, 1 \mathrm{C}, \mathrm{C}_{\mathrm{Ar}}-\mathrm{OCH}_{3}\right), 17.1\left(\mathrm{~s}, 1 \mathrm{C}, \mathrm{CH}_{3}\right) \mathrm{ppm}$.

2-Methoxy-3-methylphenol (4) A $250 \mathrm{~cm}^{3}$ three-necked round-bottom flask with a gas adapter, reflux condenser, bubbler, and a magnetic stir bar was charged with a solution of 8.20 g 1-iodo-2-methoxy-3-methylbenzene (3,33.1 mmol, $1.0 \mathrm{eq})$ in $50 \mathrm{~cm}^{3}$ DMSO. To the brown solution were subsequently added $50 \mathrm{~cm}^{3} \mathrm{NaOH}$ solution $\left(4.0 \mathrm{~mol} / \mathrm{dm}^{3}\right.$ in $\mathrm{H}_{2} \mathrm{O}$ ), $3.02 \mathrm{~g}$ glycolic acid (39.7 mmol, $\left.1.2 \mathrm{eq}\right)$ and $1.29 \mathrm{~g}$ copper(II) hydroxide (13.2 mmol, $0.4 \mathrm{eq})$. The suspension was stirred under nitrogen atmosphere for $64 \mathrm{~h}$ at $120{ }^{\circ} \mathrm{C}$ (oil bath). After cooling to $23{ }^{\circ} \mathrm{C}$ the dark brown reaction mixture was diluted with $100 \mathrm{~cm}^{3} \mathrm{H}_{2} \mathrm{O}$, acidified with $6 \mathrm{M} \mathrm{HCl}$ to $\mathrm{pH}=3$, transferred to a $500 \mathrm{~cm}^{3}$ separation funnel and extracted with EtOAc $\left(4 \times 100 \mathrm{~cm}^{3}\right)$. The combined organic phases were washed with half-saturated $\mathrm{NaCl}$ solution (200 $\left.\mathrm{cm}^{3}\right)$ and brine $\left(2 \times 200 \mathrm{~cm}^{3}\right)$, dried over $\mathrm{Na}_{2} \mathrm{SO}_{4}$, filtered, and the solvent was carefully removed under reduced pressure $\left(T \leq 30{ }^{\circ} \mathrm{C}\right)$. The crude product $(6.83 \mathrm{~g})$ was purified via flash column chromatography $\left(410 \mathrm{~g} \mathrm{SiO}_{2}\right.$; cyclohexane/ EtOAc $=10: 1(\mathrm{v} / \mathrm{v}))$ and $3.22 \mathrm{~g}(23.3 \mathrm{mmol}, 70 \%) 4$ were isolated as brown oil. $R_{f}=0.20$ (cyclohexane/EtOAc $=10: 1$ (v/v); UV/ $\mathrm{KMnO}_{4}$ ); ${ }^{1} \mathrm{H}$ NMR (300 MHz, $\mathrm{CDCl}_{3}$ ): $\delta=6.93$ (t, $J=7.8 \mathrm{~Hz}, 1 \mathrm{H}, \mathrm{Ar}-\mathrm{H}), 6.82(\mathrm{~d}, J=6.8 \mathrm{~Hz}, 1 \mathrm{H}, \mathrm{Ar}-\mathrm{H})$, $6.71(\mathrm{~d}, J=7.4 \mathrm{~Hz}, 1 \mathrm{H}, \mathrm{Ar}-\mathrm{H}), 5.76$ (bs, $1 \mathrm{H}, \mathrm{OH}), 3.81$ (s, $3 \mathrm{H}, \mathrm{OCH} 3), 2.32\left(\mathrm{~s}, 3 \mathrm{H}, \mathrm{CH}_{3}\right) \mathrm{ppm} ;{ }^{13} \mathrm{C} \mathrm{NMR}(75.5 \mathrm{MHz}$, $\left.\mathrm{CDCl}_{3}\right): \delta=149.0\left(\underline{\mathrm{C}}_{\mathrm{Ar}}-\mathrm{OH}\right), 145.6\left(\underline{\mathrm{C}}_{\mathrm{Ar}}-\mathrm{OCH}_{3}\right), 131.0$ $\left(\underline{\mathrm{C}}_{\mathrm{Ar}}-\mathrm{CH}_{3}\right), 124.7\left(\mathrm{C}_{\mathrm{Ar}}-\mathrm{H}\right), 122.6\left(\mathrm{C}_{\mathrm{Ar}}-\mathrm{H}\right), 113.3\left(\mathrm{C}_{\mathrm{Ar}}-\mathrm{H}\right)$, $60.7\left(\mathrm{OCH}_{3}\right), 15.9\left(\mathrm{CH}_{3}\right) \mathrm{ppm}$.

\section{2-Hydroxy-3-methoxy-4-methylbenzalydehyde (5) An} inert $250 \mathrm{~cm}^{3}$ three-necked round-bottom flask equipped with reflux condenser, bubbler, Schlenk line adapter, and magnetic stirring bar was charged with $3.29 \mathrm{~g}$ anhydrous $\mathrm{MgCl}_{2}$ (34.5 mmol, $1.5 \mathrm{eq}$ ) and $4.70 \mathrm{~g}$ paraformaldehyde (156 mmol, $6.8 \mathrm{eq}$ ), which were then dried for $90 \mathrm{~min}$ in oilpump vacuum. Then $60 \mathrm{~cm}^{3}$ abs. MeCN, $3.18 \mathrm{~g} 2$-methoxy3-methylphenol (4, $23.0 \mathrm{mmol}, 1.0 \mathrm{eq})$, and $12.1 \mathrm{~cm}^{3}$ abs. triethylamine ( $87.4 \mathrm{mmol}, 3.8 \mathrm{eq})$ were added and the reaction mixture was stirred for $19 \mathrm{~h}$ at $90{ }^{\circ} \mathrm{C}$ in an oil bath. After full conversion $50 \mathrm{~cm}^{3} \mathrm{H}_{2} \mathrm{SO}_{4}\left(10 \%\right.$ in $\left.\mathrm{H}_{2} \mathrm{O}\right)$ were added to the yellow suspension, and the mixture was transferred into a separation funnel and extracted with $\mathrm{CH}_{2} \mathrm{Cl}_{2}\left(4 \times 90 \mathrm{~cm}^{3}\right)$. The combined organic phases were dried over $\mathrm{Na}_{2} \mathrm{SO}_{4}$, filtered, and the solvent was carefully removed under reduced pressure $\left(T \leq 30^{\circ} \mathrm{C}\right)$. The crude product $(4.18 \mathrm{~g})$ was purified via flash column chromatography $\left(420 \mathrm{~g} \mathrm{SiO}_{2}\right.$; cyclohexane/EtOAc $=20: 1(\mathrm{v} / \mathrm{v}))$ to yield $1.84 \mathrm{~g}(11.1 \mathrm{mmol}, 48 \%)$ of compound 6 as a pale brown oil. $R_{f}=0.46$ (cyclohexane/EtOAc $=10: 1(\mathrm{v} / \mathrm{v}) ; \mathrm{UV} / \mathrm{CAM}) ;{ }^{1} \mathrm{H}$ NMR $(300 \mathrm{MHz}$, $\left.\mathrm{CDCl}_{3}\right): \delta=11.14(\mathrm{~s}, 1 \mathrm{H}, \mathrm{OH}), 9.82(\mathrm{~s}, 1 \mathrm{H}, \mathrm{CHO}), 7.19$ $(\mathrm{d}, J=7.9 \mathrm{~Hz}, 1 \mathrm{H}, \mathrm{Ar}-\mathrm{H}), 6.81(\mathrm{~d}, J=7.9 \mathrm{~Hz}, 1 \mathrm{H}, \mathrm{Ar}-\mathrm{H})$, $3.89\left(\mathrm{~s}, 3 \mathrm{H}, \mathrm{OCH}_{3}\right), 2.33\left(\mathrm{~s}, 3 \mathrm{H}, \mathrm{CH}_{3}\right) \mathrm{ppm} ;{ }^{13} \mathrm{C} \mathrm{NMR}$ $\left(75.5 \mathrm{MHz}, \mathrm{CDCl}_{3}\right): \delta=196.2(\mathrm{CHO}), 154.9\left(\mathrm{C}_{\mathrm{Ar}}-\mathrm{OH}\right)$, $146.3\left(\underline{\mathrm{C}}_{\mathrm{Ar}}-\mathrm{OCH}_{3}\right), 141.0\left(\underline{\mathrm{C}}_{\mathrm{Ar}}-\mathrm{CH}_{3}\right), 128.2\left(\mathrm{~s}, 1 \mathrm{C}, \mathrm{C}_{\mathrm{Ar}}-\mathrm{H}\right)$, $121.9\left(\mathrm{C}_{\mathrm{Ar}}-\mathrm{H}\right), 120.4\left(\mathrm{C}_{\mathrm{Ar}}-\mathrm{CHO}\right), 60.2\left(\mathrm{OCH}_{3}\right), 16.9\left(\mathrm{CH}_{3}\right)$ ppm.

\section{2-Methoxy-3-methyl-6-[(methylamino)methyl]phenol}

(6a) An inert $25 \mathrm{~cm}^{3}$ Schlenk tube with magnetic stirring bar was charged with a solution of $535 \mathrm{mg}$ 2-hydroxy3-methoxy-4-methylbenzalydehyde (5, $3.22 \mathrm{mmol}, 1.0 \mathrm{eq})$ in $4.0 \mathrm{~cm}^{3}$ abs. $\mathrm{MeOH}$ and $441 \mathrm{~mm}^{3}$ methylamine solution (33\% in abs. EtOH, $3.54 \mathrm{mmol}, 1.1 \mathrm{eq}$ ) were added. The yellow solution was stirred for $60 \mathrm{~min}$ at $23{ }^{\circ} \mathrm{C}$, and after full conversion (reaction control via TLC; cyclohexane/ $\left.\mathrm{EtOAc}=10: 1(\mathrm{v} / \mathrm{v}) ; R_{f}=0.10 ; \mathrm{UV} / \mathrm{CAM}\right)$ the reaction mixture was cooled to $0{ }^{\circ} \mathrm{C}$ (ice bath) and treated with $122 \mathrm{mg}$ $\mathrm{NaBH}_{4}(3.22 \mathrm{mmol}, 1.0 \mathrm{eq})$. The ice bath was removed and the pale yellow solution was stirred at $23{ }^{\circ} \mathrm{C}$ for additional $45 \mathrm{~min}$. Subsequently, the volatiles were removed under reduced pressure. The colorless residue was partitioned between $15 \mathrm{~cm}^{3}$ EtOAc and $15 \mathrm{~cm}^{3} \mathrm{H}_{2} \mathrm{O}$ in a $50 \mathrm{~cm}^{3}$ separation funnel and the aqueous phase was extracted with EtOAc $\left(4 \times 15 \mathrm{~cm}^{3}\right)$. The combined organic phases were dried over $\mathrm{Na}_{2} \mathrm{SO}_{4}$, filtered, and the solvent was removed under reduced pressure. The crude product $(566 \mathrm{mg})$ was dried in oil-pump vacuum and purified via flash column chromatography $\left(57 \mathrm{~g} \mathrm{SiO}_{2} ; \mathrm{CH}_{2} \mathrm{Cl}_{2} / \mathrm{MeOH}=15: 1+1 \% \mathrm{NH}_{4} \mathrm{OH}\right.$ $(\mathrm{v} / \mathrm{v}))$ to yield $501 \mathrm{mg}(2.76 \mathrm{mmol}, 86 \%)$ of $\mathbf{6 a}$ as a pale brown oil. $R_{f}=0.20\left(\mathrm{CH}_{2} \mathrm{Cl}_{2} / \mathrm{MeOH}=15: 1+1 \% \mathrm{NH}_{4} \mathrm{OH}\right.$ (v/v); UV/KMnO $\left.{ }_{4}\right) ;{ }^{1} \mathrm{H}$ NMR $\left(300 \mathrm{MHz}, \mathrm{CDCl}_{3}\right): \delta=6.64$ (d, $J=7.6 \mathrm{~Hz}, 1 \mathrm{H}, \mathrm{Ar}-\mathrm{H}), 6.57$ (d, $J=7.6 \mathrm{~Hz}, 1 \mathrm{H}, \mathrm{Ar}-\mathrm{H})$, $3.94\left(\mathrm{~s}, 2 \mathrm{H}, \mathrm{CH}_{2}\right), 3.86$ (s, 3H, $\left.\mathrm{OCH}_{3}\right), 2.47$ (s, $3 \mathrm{H}, \mathrm{NCH}_{3}$ ), $2.25\left(\mathrm{~s}, 3 \mathrm{H}, \mathrm{C}_{\mathrm{Ar}}-\mathrm{CH}_{3}\right) \mathrm{ppm} ;{ }^{13} \mathrm{C} \mathrm{NMR}\left(75.5 \mathrm{MHz}, \mathrm{CDCl}_{3}\right)$ : $\delta=151.3\left(\underline{\mathrm{C}}_{\mathrm{Ar}}-\mathrm{OCH} 3\right), 146.3\left(\underline{\mathrm{C}}_{\mathrm{Ar}}-\mathrm{OH}\right), 131.0\left(\underline{\mathrm{C}}_{\mathrm{Ar}}-\mathrm{CH}_{3}\right)$, $123.0\left(\mathrm{C}_{\mathrm{Ar}}-\mathrm{H}\right), 121.3\left(\underline{\mathrm{C}}_{\mathrm{Ar}}-\mathrm{CH}_{2}\right), 120.2\left(\mathrm{C}_{\mathrm{Ar}}-\mathrm{H}\right), 59.9$ $\left(\mathrm{OCH}_{3}\right), 54.4\left(\mathrm{CH}_{2}\right), 35.1\left(\mathrm{NCH}_{3}\right), 15.9\left(\mathrm{C}_{\mathrm{Ar}}-\mathrm{CH}_{3}\right) \mathrm{ppm}$.

6-[[(2,2-Diethoxyethyl)amino]methyl]-2-methoxy-3-methylphenol $\left(6, \mathrm{C}_{15} \mathrm{H}_{25} \mathrm{NO}_{4}\right)$ An inert $50 \mathrm{~cm}^{3}$ round-bottom flask with Schlenk adapter and magnetic stirring bar was charged with a solution of $1.78 \mathrm{~g}$ 
2-hydroxy-3-methoxy-4-methylbenzalydehyde (5, $10.7 \mathrm{mmol}, 1.0 \mathrm{eq})$ in $13 \mathrm{~cm}^{3}$ abs. $\mathrm{MeOH}$ and $1.86 \mathrm{~cm}^{3}$ aminoacetaldehyde diethyl acetal $(12.9 \mathrm{mmol}, 1.2 \mathrm{eq})$ were added. The yellow solution was stirred for $30 \mathrm{~min}$ at $23{ }^{\circ} \mathrm{C}$, and after full conversion (reaction control via TLC; cyclohexane/EtOAc $\left.=10: 1(\mathrm{v} / \mathrm{v}) ; R_{f}=0.17 ; \mathrm{UV} / \mathrm{KMnO}_{4}\right)$ the reaction mixture was cooled to $0{ }^{\circ} \mathrm{C}$ (ice bath) and treated portionwise with $0.61 \mathrm{~g} \mathrm{NaBH}_{4}(16.1 \mathrm{mmol}, 1.5 \mathrm{eq})$. After completion of the gas evolution $(\sim 5 \mathrm{~min})$ the ice bath was removed and the pale yellow solution was stirred at $23{ }^{\circ} \mathrm{C}$ for additional $30 \mathrm{~min}$. Subsequently, the volatiles were removed under reduced pressure. The yellow residue was partitioned between $40 \mathrm{~cm}^{3}$ EtOAc and $20 \mathrm{~cm}^{3} \mathrm{H}_{2} \mathrm{O}$ in a $100 \mathrm{~cm}^{3}$ separation funnel and the aqueous phase was extracted with EtOAc $\left(2 \times 20 \mathrm{~cm}^{3}\right)$. The combined organic phases were dried over $\mathrm{Na}_{2} \mathrm{SO}_{4}$, filtered, and the solvent was removed under reduced pressure. After drying for $3 \mathrm{~h}$ in oil-pump vacuum, the red-brown oil (3.03 g, $10.7 \mathrm{mmol}$, quant.) was directly used in the next step without further purification. $R_{f}=0.08($ cyclohexane/EtOAc $=5: 1(\mathrm{v} / \mathrm{v})$; $\left.\mathrm{UV} / \mathrm{KMnO}_{4}\right) ;{ }^{1} \mathrm{H}$ NMR $\left(300 \mathrm{MHz}, \mathrm{CDCl}_{3}\right): \delta=6.64(\mathrm{~d}$, $J=7.6 \mathrm{~Hz}, 1 \mathrm{H}, \mathrm{Ar}-\mathrm{H}), 6.57(\mathrm{~d}, J=7.7 \mathrm{~Hz}, 1 \mathrm{H}, \mathrm{Ar}-\mathrm{H}), 4.61$ (t, $\left.J=5.5 \mathrm{~Hz}, 1 \mathrm{H}, \mathrm{CH}_{2} \mathrm{C} \underline{\mathrm{H}}\right), 3.98\left(\mathrm{~s}, 2 \mathrm{H}, \mathrm{C}_{\mathrm{Ar}}-\mathrm{C}_{2} \mathrm{NH}\right), 3.85$ (s, 3H, $\mathrm{OCH}_{3}$ ), 3.75-3.62 (m, 2H, $\left.\mathrm{CH}_{2} \mathrm{CH}_{3}\right), 3.61-3.43$ (m, $2 \mathrm{H}, \mathrm{CH}_{2} \mathrm{CH}_{3}$ '), 2.77 (d, J=5.5 Hz, 2H, $\mathrm{CH}_{2} \mathrm{CH}$ ), 2.24 (s, $3 \mathrm{H}, \mathrm{C}_{\mathrm{Ar}}-\underline{\mathrm{CH}}_{3}$ ), 1.20 (t, $\left.J=7.0 \mathrm{~Hz}, 6 \mathrm{H}, 2 \times \mathrm{CH}_{2} \mathrm{C}_{3}\right) \mathrm{ppm}$; ${ }^{13} \mathrm{C}$ NMR $\left(75.5 \mathrm{MHz}, \mathrm{CDCl}_{3}\right): \delta=151.2\left(\underline{\mathrm{C}}_{\mathrm{Ar}}-\mathrm{OCH} 3\right)$, $146.4\left(\mathrm{C}_{\mathrm{Ar}}-\mathrm{OH}\right), 131.3\left(\mathrm{C}_{\mathrm{Ar}}-\mathrm{CH}_{3}\right), 123.1\left(\mathrm{C}_{\mathrm{Ar}}-\mathrm{H}\right), 121.4$ $\left(\underline{\mathrm{C}}_{\mathrm{Ar}}-\mathrm{CH}_{2}\right), 120.5\left(\mathrm{C}_{\mathrm{Ar}}-\mathrm{H}\right), 101.6\left(\mathrm{CH}_{2} \mathrm{CH}\right), 62.9(2 \mathrm{C}$, $\left.2 \times \mathrm{CH}_{2} \mathrm{CH}_{3}\right), 60.0\left(\mathrm{OCH}_{3}\right), 52.3\left(\mathrm{C}_{\mathrm{Ar}}-\mathrm{CH}_{2}\right), 50.8\left(\mathrm{CH}_{2} \mathrm{CH}\right)$, $16.0\left(\mathrm{C}_{\mathrm{Ar}}-\mathrm{CH}_{3}\right), 15.5\left(2 \mathrm{C}, 2 \times \mathrm{CH}_{2} \mathrm{CH}_{3}\right)$ ppm; HR-ESI-MS: calcd. $m / z$ for $\left[\mathrm{C}_{15} \mathrm{H}_{25} \mathrm{NO}_{4}+\mathrm{H}\right]^{+} 284.1856$, found 284.1857.

N-(2,2-Diethoxyethyl)-N-(2-hydroxy-3-methoxy-4-methylbenzyl)-4-methylbenzenesulfonamide (7, $\mathrm{C}_{22} \mathrm{H}_{31} \mathrm{NO}_{6} \mathrm{~S}$ ) An inert $50 \mathrm{~cm}^{3}$ round-bottom flask with Schlenk adapter and magnetic stirring bar was charged with a solution of $2.75 \mathrm{~g}$ compound $6(9.70 \mathrm{mmol}, 1.0 \mathrm{eq})$ in $13 \mathrm{~cm}^{3}$ abs. $\mathrm{CH}_{2} \mathrm{Cl}_{2}$. Then $1.94 \mathrm{~g} p$-toluenesulfonic acid (10.2 mmol, $1.05 \mathrm{eq})$ and $0.81 \mathrm{~g}$ sodium bicarbonate $(9.7 \mathrm{mmol}, 1.0 \mathrm{eq})$ were added to the solution, the flask was equipped with a bubbler, and the reaction mixture was stirred at $23{ }^{\circ} \mathrm{C}$ for $24 \mathrm{~h}$, after which time the volatiles were removed under reduced pressure. The crude product $(4.91 \mathrm{~g}$ ) was purified via flash column chromatography $\left(500 \mathrm{~g} \mathrm{SiO}_{2}\right.$; cyclohexane/EtOAc $=3: 1(\mathrm{v} / \mathrm{v}))$ to give the desired product ( $3.95 \mathrm{~g}, 9.03 \mathrm{mmol}, 93 \%)$ as a colorless oil. $R_{f}=0.42$ $\left(\right.$ cyclohexane $\left./ \mathrm{EtOAc}=3: 1(\mathrm{v} / \mathrm{v}) ; \mathrm{UV} / \mathrm{KMnO}_{4}\right) ;{ }^{1} \mathrm{H}$ NMR (300 MHz, $\mathrm{CDCl}_{3}$ ): $\delta=7.68$ (d, $\left.J=8.2 \mathrm{~Hz}, 2 \mathrm{H}, \mathrm{Ts}-\mathrm{H}\right), 7.26$ (d, $J=8.6 \mathrm{~Hz}, 2 \mathrm{H}$, Ts-H), $6.84(\mathrm{~d}, J=7.8 \mathrm{~Hz}, 1 \mathrm{H}, \mathrm{Ar}-\mathrm{H})$, $6.62(\mathrm{~d}, J=7.9 \mathrm{~Hz}, 1 \mathrm{H}, \mathrm{Ar}-\mathrm{H}), 6.32(\mathrm{~s}, 1 \mathrm{H}, \mathrm{OH}), 4.60$ (t, $\left.J=5.4 \mathrm{~Hz}, 1 \mathrm{H}, \mathrm{CH}_{2} \mathrm{C} \underline{\mathrm{H}}\right), 4.49\left(\mathrm{~s}, 2 \mathrm{H}, \mathrm{C}_{\mathrm{Ar}}-\mathrm{C}_{2} \mathrm{~N}\right), 3.76(\mathrm{~s}$, $\left.3 \mathrm{H}, \mathrm{OCH}_{3}\right), 3.67-3.62\left(\mathrm{~m}, 2 \mathrm{H}, \mathrm{C}_{2} \mathrm{CH}_{3}\right), 3.52-3.35(\mathrm{~m}$,
$2 \mathrm{H}, \mathrm{C}_{2} \mathrm{CH}_{3}$ '), 3.31 (d, J=5.4 Hz, 2H, $\underline{\mathrm{H}}_{2} \mathrm{CH}$ ), 2.42 (s, 3H, $\mathrm{C}_{\mathrm{Ar}}-\mathrm{C}_{3}$ of Ts), 2.26 (s, 3H, $\left.\mathrm{C}_{\mathrm{Ar}}-\mathrm{C}_{3}\right), 1.14$ (t, $\left.J=7.0 \mathrm{~Hz}, 6 \mathrm{H}, 2 \times \mathrm{CH}_{2} \mathrm{CH}_{3}\right) \mathrm{ppm} ;{ }^{13} \mathrm{C} \mathrm{NMR}(75.5 \mathrm{MHz}$, $\left.\mathrm{CDCl}_{3}\right): \delta=147.8\left(\underline{\mathrm{C}}_{\mathrm{Ar}}-\mathrm{OCH}_{3}\right), 145.9\left(\mathrm{C}_{\mathrm{Ar}}-\mathrm{OH}\right), 143.2$ $\left(\mathrm{C}-\mathrm{SO}_{2}\right), 137.5\left(\underline{\mathrm{C}}_{\mathrm{Ar}}-\mathrm{CH}_{3}\right.$ of Ts$), 130.9\left(\underline{\mathrm{C}}_{\mathrm{Ar}}-\mathrm{CH}_{3}\right), 129.6$ $\left(2 \mathrm{C}, \mathrm{C}_{\mathrm{Ar}}-\mathrm{H}\right.$ of Ts), $127.4\left(2 \mathrm{C}, \mathrm{C}_{\mathrm{Ar}}-\mathrm{H}\right.$ of Ts), $125.8\left(\mathrm{C}_{\mathrm{Ar}}-\mathrm{H}\right)$, $121.8\left(\mathrm{C}_{\mathrm{Ar}}-\mathrm{H}\right), 120.3\left(\underline{\mathrm{C}}_{\mathrm{Ar}}-\mathrm{CH}_{2}\right), 102.1\left(\mathrm{CH}_{2} \underline{\mathrm{CH}}\right), 63.4(2 \mathrm{C}$, $\left.2 \times \underline{\mathrm{CH}}_{2} \mathrm{CH}_{3}\right), 60.5\left(\mathrm{OCH}_{3}\right), 50.0\left(\underline{\mathrm{CH}}_{2} \mathrm{CH}\right), 48.0\left(\mathrm{C}_{\mathrm{Ar}}-\underline{\mathrm{C}} \mathrm{H}_{2}\right)$, $21.6\left(\mathrm{CH}_{3}\right.$ of Ts), $15.9\left(\mathrm{CH}_{3}\right), 15.3\left(2 \mathrm{C}, \mathrm{CH}_{2} \mathrm{CH}_{3}\right) \mathrm{ppm}$; HRESI-MS: calcd. $m / z$ for $\left[\mathrm{C}_{22} \mathrm{H}_{31} \mathrm{NO}_{6} \mathrm{~S}-\mathrm{H}\right]^{-} 436.1799$, found 436.1804 .

$\mathrm{N}$-[2-(Benzyloxy)-3-methoxy-4-methylbenzyl]-N-(2,2-diethoxyethyl)-4-methylbenzenesulfonamide $\left(8, \mathrm{C}_{29} \mathrm{H}_{37} \mathrm{NO}_{6} \mathrm{~S}\right)$ An inert $100 \mathrm{~cm}^{3}$ round-bottom flask with Schlenk adapter and magnetic stirring bar was charged with a solution of $3.79 \mathrm{~g} 7$ (8.66 mmol, $1.0 \mathrm{eq})$ in $43 \mathrm{~cm}^{3}$ abs. DMF. Then $1.80 \mathrm{~g}$ potassium carbonate $(13.0 \mathrm{mmol}, 1.5 \mathrm{eq})$ and 1.54 $\mathrm{cm}^{3}$ benzyl bromide (13.0 mmol, $1.5 \mathrm{eq}$ ) were added to the colorless solution and the suspension was stirred at $40{ }^{\circ} \mathrm{C}$ (oil bath) overnight. The reaction mixture was transferred into a $250 \mathrm{~cm}^{3}$ separation funnel, diluted with $130 \mathrm{~cm}^{3}$ EtOAc, and washed with $\mathrm{H}_{2} \mathrm{O}\left(5 \times 90 \mathrm{~cm}^{3}\right)$ and brine $(90$ $\mathrm{cm}^{3}$ ). The organic phase was dried over $\mathrm{Na}_{2} \mathrm{SO}_{4}$, filtered, and the solvent was removed under reduced pressure. The crude product $(4.75 \mathrm{~g})$ was purified via flash column chromatography $\left(475 \mathrm{~g} \mathrm{SiO}_{2}\right.$; cyclohexane/EtOAc $\left.=7: 1(\mathrm{v} / \mathrm{v})\right)$ to yield $4.25 \mathrm{~g}$ (8.05 mmol, 93\%) of $\mathbf{8}$ as a colorless oil. $R_{f}=0.31\left(\right.$ cyclohexane/EtOAc $\left.=7: 1(\mathrm{v} / \mathrm{v}) ; \mathrm{UV} / \mathrm{KMnO}_{4}\right)$; ${ }^{1} \mathrm{H}$ NMR $\left(300 \mathrm{MHz}, \mathrm{CDCl}_{3}\right): \delta=7.63(\mathrm{~d}, J=8.2 \mathrm{~Hz}, 2 \mathrm{H}$, Ts-H), 7.47-7.28 (m, 5H, Bn-H), $7.23(\mathrm{~d}, J=8.2 \mathrm{~Hz}, 2 \mathrm{H}$, Ts-H), 6.95 (d, J=7.9 Hz, 1H, Ar-H), 6.85 (d, $J=7.9 \mathrm{~Hz}$, $1 \mathrm{H}, \mathrm{Ar}-\mathrm{H}), 4.98\left(\mathrm{~s}, 2 \mathrm{H}, \mathrm{Bn}-\mathrm{CH}_{2}\right), 4.52(\mathrm{t}, J=5.4 \mathrm{~Hz}, 1 \mathrm{H}$, $\left.\mathrm{CH}_{2} \mathrm{CH}\right), 4.42\left(\mathrm{~s}, 2 \mathrm{H}, \mathrm{C}_{\mathrm{Ar}}-\mathrm{CH}_{2} \mathrm{~N}\right), 3.78\left(\mathrm{~s}, 3 \mathrm{H}, \mathrm{OCH}_{3}\right)$, 3.65-3.45 (m, $2 \mathrm{H}, \mathrm{CH}_{2} \mathrm{CH}_{3}$ ), 3.43-3.26 (m, $2 \mathrm{H}, \mathrm{CH}_{2} \mathrm{CH}_{3}$ ) ), $3.22\left(\mathrm{~d}, J=5.4 \mathrm{~Hz}, 2 \mathrm{H}, \mathrm{CH}_{2} \mathrm{CH}\right), 2.41\left(\mathrm{~s}, 3 \mathrm{H}, \mathrm{CH}_{3}\right.$ of Ts), $2.26\left(\mathrm{~s}, 3 \mathrm{H}, \mathrm{CH}_{3}\right), 1.05\left(\mathrm{t}, J=7.0 \mathrm{~Hz}, 6 \mathrm{H}, 2 \times \mathrm{CH}_{2} \mathrm{CH}_{3}\right) \mathrm{ppm}$; ${ }^{13} \mathrm{C} \mathrm{NMR}\left(75.5 \mathrm{MHz}, \mathrm{CDCl}_{3}\right): \delta=151.4\left(\underline{\mathrm{C}}_{\mathrm{Ar}}-\mathrm{OCH}_{3}\right), 149.9$ $\left(\underline{\mathrm{C}}_{\mathrm{Ar}}-\mathrm{OBn}\right), 143.1\left(\mathrm{C}-\mathrm{SO}_{2}\right), 137.7\left(\mathrm{Bn}-\mathrm{C}_{\mathrm{q}}\right), 137.6\left(\underline{\mathrm{C}}_{\mathrm{Ar}}-\mathrm{CH}_{3}\right.$ of Ts), $131.8\left(\underline{\mathrm{C}}_{\mathrm{Ar}}-\mathrm{CH}_{3}\right), 129.6\left(2 \mathrm{C}, \mathrm{C}_{\mathrm{Ar}}-\mathrm{H}\right.$ of Ts $), 128.7$ $\left(\mathrm{C}_{\mathrm{q}}-\mathrm{Ar}\right), 128.5\left(2 \mathrm{C}, \mathrm{C}_{\mathrm{Ar}}-\mathrm{H}\right.$ of $\left.\mathrm{Bn}\right), 128.4\left(2 \mathrm{C}, \mathrm{C}_{\mathrm{Ar}}-\mathrm{H}\right.$ of $\left.\mathrm{Bn}\right)$, $128.1\left(\mathrm{C}_{\mathrm{q}}-\mathrm{Ar}\right), 127.4\left(2 \mathrm{C}, \mathrm{C}_{\mathrm{Ar}}-\mathrm{H}\right.$ of Ts$), 125.9\left(\mathrm{C}_{\mathrm{Ar}}-\mathrm{H}\right)$, $124.4\left(\mathrm{C}_{\mathrm{Ar}}-\mathrm{H}\right), 102.0\left(\mathrm{CH}_{2} \underline{\mathrm{CH}}\right), 74.9\left(\mathrm{Bn}-\mathrm{CH}_{2}\right), 63.1(2 \mathrm{C}$, $\left.2 \times \mathrm{CH}_{2} \mathrm{CH}_{3}\right), 60.3\left(\mathrm{OCH}_{3}\right), 50.7\left(\mathrm{CH}_{2} \mathrm{CH}\right), 47.7\left(\mathrm{C}_{\mathrm{Ar}}-\mathrm{CH}_{2}\right)$, $21.6\left(\mathrm{CH}_{3}\right.$ of Ts), $15.9\left(\mathrm{CH}_{3}\right), 15.3\left(2 \mathrm{C}, 2 \times \mathrm{CH}_{2} \mathrm{CH}_{3}\right) \mathrm{ppm}$; HR-ESI-MS: calcd. $m / z$ for $\left[\mathrm{C}_{29} \mathrm{H}_{37} \mathrm{NO}_{6} \mathrm{~S}+\mathrm{NH}_{4}\right]^{+} 545.2680$, found 545.2682.

8-(Benzyloxy)-7-methoxy-6-methyl-2-tosyl-1,2-dihydroisoquinoline $\left(\mathbf{9}, \mathrm{C}_{\mathbf{2 5}} \mathrm{H}_{\mathbf{2 5}} \mathrm{NO}_{\mathbf{4}} \mathrm{S}\right)$ A $250 \mathrm{~cm}^{3}$ round-bottom flask with reflux condenser and magnetic stirring bar was charged with a solution of $4.13 \mathrm{~g} 8(7.82 \mathrm{mmol}, 1.0 \mathrm{eq})$ in $80 \mathrm{~cm}^{3}$ 1,4-dioxane. After addition of $6.5 \mathrm{~cm}^{3} 6 \mathrm{M} \mathrm{HCl}$ 
(39 mmol, 5.0 eq) the mixture was heated to $110{ }^{\circ} \mathrm{C}$ (oil bath) and heated under reflux for $6.5 \mathrm{~h}$. The brown solution was cooled to $23{ }^{\circ} \mathrm{C}$, the solvent removed under reduced pressure, and the crude residue ( $3.95 \mathrm{~g}$ ) dried in oil-pump vacuum. Purification via flash column chromatography (475 $\mathrm{g} \mathrm{SiO}_{2}$, cyclohexane/EtOAc $\left.=10: 1(\mathrm{v} / \mathrm{v})\right)$ provided $1.65 \mathrm{~g}(3.79 \mathrm{mmol}, 48 \%) 9$ as a colorless solid. $R_{f}=0.27$ $\left(\right.$ cyclohexane/EtOAc $\left.=10: 1(\mathrm{v} / \mathrm{v}) ; \mathrm{UV} / \mathrm{KMnO}_{4}\right) ;$ m.p.: 104$109{ }^{\circ} \mathrm{C} ;{ }^{1} \mathrm{H}$ NMR $\left(300 \mathrm{MHz}, \mathrm{CDCl}_{3}\right): \delta=7.64(\mathrm{~d}, J=8.2 \mathrm{~Hz}$, $2 \mathrm{H}, \mathrm{Ts}-\mathrm{H}), 7.50-7.30(\mathrm{~m}, 5 \mathrm{H}, \mathrm{Bn}-\mathrm{H}), 7.25(\mathrm{~d}, J=8.1 \mathrm{~Hz}$, $2 \mathrm{H}, \mathrm{Ts}-\mathrm{H}), 6.69(\mathrm{~d}, J=7.7 \mathrm{~Hz}, 1 \mathrm{H}, \mathrm{NCH}=\mathrm{CH}), 6.54(\mathrm{~s}, 1 \mathrm{H}$, $\mathrm{Ar}-\mathrm{H}), 5.72(\mathrm{~d}, J=7.8 \mathrm{~Hz}, 1 \mathrm{H}, \mathrm{NCH}=\mathrm{CH}), 4.94(\mathrm{~s}, 2 \mathrm{H}, \mathrm{Bn}-$ $\mathrm{CH}_{2}$ ), 4.44 (s, $\left.2 \mathrm{H}, \mathrm{NCH}_{2}\right), 3.80$ (s, $\left.3 \mathrm{H}, \mathrm{OCH}_{3}\right), 2.39$ (s, $3 \mathrm{H}$, $\mathrm{CH}_{3}$ of Ts), $2.20\left(\mathrm{~s}, 3 \mathrm{H}, \mathrm{CH}_{3}\right) \mathrm{ppm} ;{ }^{13} \mathrm{C}$ NMR $(75.5 \mathrm{MHz}$, $\left.\mathrm{CDCl}_{3}\right): \delta=150.9\left(\underline{\mathrm{C}}_{\mathrm{Ar}}-\mathrm{OCH}_{3}\right), 147.6\left(\underline{\mathrm{C}}_{\mathrm{Ar}}-\mathrm{OBn}\right), 144.0\left(\mathrm{C}_{\mathrm{q}}\right.$ of Ts), $137.3\left(\mathrm{C}_{\mathrm{q}}\right.$ of $\left.\mathrm{Bn}\right), 134.9\left(\mathrm{CSO}_{2}\right), 131.6\left(\underline{\mathrm{C}}_{\mathrm{Ar}}-\mathrm{CH}_{3}\right)$, $129.8\left(2 \mathrm{C}, \mathrm{C}_{\mathrm{Ar}}-\mathrm{H}\right.$ of Ts$), 128.7\left(2 \mathrm{C}, \mathrm{C}_{\mathrm{Ar}}-\mathrm{H}\right.$ of $\left.\mathrm{Bn}\right), 128.6$ $\left(2 \mathrm{C}, \mathrm{C}_{\mathrm{Ar}}-\mathrm{H}\right.$ of $\left.\mathrm{Bn}\right), 128.4\left(\mathrm{C}_{\mathrm{Ar}}-\mathrm{H}\right.$ of $\left.\mathrm{Bn}\right), 127.3\left(2 \mathrm{C}, \mathrm{C}_{\mathrm{Ar}}-\mathrm{H}\right.$ of Ts), $126.7\left(\mathrm{C}_{\mathrm{q}}-\mathrm{Ar}\right), 125.6(\mathrm{NCH}=\mathrm{CH}), 122.3\left(\underline{\mathrm{C}}_{\mathrm{Ar}}-\mathrm{H}\right)$, $119.7\left(\mathrm{C}_{\mathrm{q}}-\mathrm{Ar}\right), 109.6(\mathrm{NCH}=\underline{\mathrm{CH}}), 75.2\left(\mathrm{Bn}-\mathrm{CH}_{2}\right), 60.4$ $\left(\mathrm{OCH}_{3}\right), 42.2\left(\mathrm{NCH}_{2}\right), 21.6\left(\mathrm{CH}_{3}\right.$ of $\left.\mathrm{Ts}\right), 15.8\left(\mathrm{CH}_{3}\right) \mathrm{ppm}$; HR-ESI-MS: calcd. $m / z$ for $\left[\mathrm{C}_{25} \mathrm{H}_{25} \mathrm{NO}_{4} \mathrm{~S}+\mathrm{H}\right]^{+} 436.1577$, found 436.1579 .

\section{8-(Benzyloxy)-7-methoxy-6-methylisoquinoline (10,} $\left.\mathbf{C}_{\mathbf{1 8}} \mathbf{H}_{\mathbf{1 7}} \mathbf{N O}_{\mathbf{2}}\right)$ A $50 \mathrm{~cm}^{3}$ round-bottom flask containing $1.65 \mathrm{~g}$ 9 (3.79 mmol, 1.0 eq) was equipped with a Schlenk adapter and magnetic stirring bar and purged with $\operatorname{Ar}(3 \times)$. The solid was partially dissolved in warm $t$ - BuOH $\left(15 \mathrm{~cm}^{3}\right)$ and $2.34 \mathrm{~g}$ potassium tert-butoxide $(20.9 \mathrm{mmol}, 5.5 \mathrm{eq})$ were added. The mixture was heated to $80^{\circ} \mathrm{C}$ (oil bath) and the resulting brown solution was stirred for $16 \mathrm{~h}$, after which time it was cooled to $23{ }^{\circ} \mathrm{C}$ and diluted with $30 \mathrm{~cm}^{3} \mathrm{H}_{2} \mathrm{O}$. The product was extracted with an appropriate amount of EtOAc $(3 \times)$, and the combined organic phases were washed with brine, dried over $\mathrm{Na}_{2} \mathrm{SO}_{4}$ and filtered. The solvent was removed under reduced pressure and the crude product $(988 \mathrm{mg})$ dried in oil-pump vacuum. Purification via flash column chromatography $\left(100 \mathrm{~g} \mathrm{SiO}_{2}\right.$, cyclohexane/EtOAc $=3: 1$ $(\mathrm{v} / \mathrm{v}))$ provided $925 \mathrm{mg}(3.31 \mathrm{mmol}, 87 \%) \mathbf{1 0}$ as a colorless solid. $R_{f}=0.25($ cyclohexane/EtOAc $=3: 1(\mathrm{v} / \mathrm{v}) ; \mathrm{UV} / \mathrm{CAM})$; m.p.: $68-70{ }^{\circ} \mathrm{C} ;{ }^{1} \mathrm{H}$ NMR $\left(300 \mathrm{MHz}, \mathrm{CDCl}_{3}\right): \delta=9.45$ (s, $1 \mathrm{H}, \mathrm{H}-1), 8.42$ (d, J=5.7 Hz, 1H, H-3), 7.61-7.29 (m, 7H, $\mathrm{Ar}-\mathrm{H}), 5.22\left(\mathrm{~s}, 2 \mathrm{H}, \mathrm{CH}_{2}\right), 3.99\left(\mathrm{~s}, 3 \mathrm{H}, \mathrm{OCH}_{3}\right), 2.48(\mathrm{~s}$, $\left.3 \mathrm{H}, \mathrm{CH}_{3}\right) \mathrm{ppm} ;{ }^{13} \mathrm{C}$ NMR $\left(75.5 \mathrm{MHz}, \mathrm{CDCl}_{3}\right): \delta=149.3$ $\left(\underline{\mathrm{C}}_{\mathrm{Ar}}-\mathrm{OCH}_{3}\right), 147.4(\mathrm{C}-1), 146.4\left(\underline{\mathrm{C}}_{\mathrm{Ar}}-\mathrm{OBn}\right), 142.6(\mathrm{C}-3)$, $138.2\left(\underline{\mathrm{C}}_{\mathrm{q}}-\mathrm{CH}_{3}\right), 137.1\left(\underline{\mathrm{C}}_{\mathrm{q}}-\mathrm{CH}_{2}\right), 133.4\left(\mathrm{C}_{\mathrm{q}}-\mathrm{Ar}\right), 128.8(2 \mathrm{C}$, $\mathrm{C}_{\mathrm{Ar}}-\mathrm{H}$ of $\left.\mathrm{Bn}\right), 128.6\left(2 \mathrm{C}, \mathrm{C}_{\mathrm{Ar}}-\mathrm{H}\right.$ of $\left.\mathrm{Bn}\right), 128.5\left(\mathrm{C}_{\mathrm{Ar}}-\mathrm{H}\right.$ of $\mathrm{Bn}), 123.9\left(\mathrm{C}_{\mathrm{q}}-\mathrm{Ar}\right), 122.9(\mathrm{C}-5), 119.3(\mathrm{C}-4), 75.9\left(\mathrm{CH}_{2}\right)$, $60.7\left(\mathrm{OCH}_{3}\right), 17.4\left(\mathrm{CH}_{3}\right)$ ppm; HR-ESI-MS: calcd. $\mathrm{m} / z$ for $\left[\mathrm{C}_{18} \mathrm{H}_{17} \mathrm{NO}_{2}+\mathrm{H}\right]^{+}$280.1332, found 280.1334 .
7-Methoxy-6-methylisoquinolin-8-ol (11, $\mathbf{C}_{\mathbf{1 1}} \mathbf{H}_{\mathbf{1 1}} \mathbf{N O}_{\mathbf{2}}$ ) Compound $\mathbf{1 0}(850 \mathrm{mg}, 3.04 \mathrm{mmol})$ was dissolved in $75 \mathrm{~cm}^{3} \mathrm{MeOH}$ and the pale yellow solution was subjected to catalytic hydrogenation using an $\mathrm{H}$-cube ${ }^{\circledR}$ continuous-flow reactor (ThalesNano Inc.) at $1.0 \mathrm{~cm}^{3} / \mathrm{min}$ and $2.8 \times 10^{5} \mathrm{~Pa}$ equipped with a $10 \% \mathrm{Pd} / \mathrm{C}$ cartridge at $30{ }^{\circ} \mathrm{C}$. The solvent was removed under reduced pressure and the crude product $(736 \mathrm{mg}$ ) was purified via flash column chromatography $\left(75 \mathrm{~g} \mathrm{SiO}_{2}, \mathrm{CH}_{2} \mathrm{Cl}_{2} / \mathrm{MeOH}=20: 1(\mathrm{v} / \mathrm{v})\right)$ to provide $318 \mathrm{mg}(1.68 \mathrm{mmol}, 55 \%)$ of $\mathbf{1 1}$ as a salmon-colored solid. $R_{f}=0.17\left(\mathrm{CH}_{2} \mathrm{Cl}_{2} / \mathrm{MeOH}=20: 1(\mathrm{v} / \mathrm{v}) ; \mathrm{UV} / \mathrm{CAM}\right)$; m.p.: $188{ }^{\circ} \mathrm{C} ;{ }^{1} \mathrm{H}$ NMR $\left(300 \mathrm{MHz}\right.$, methanol- $\left.d_{4}\right): \delta=9.37$ (s, $1 \mathrm{H}, \mathrm{H}-1), 8.24(\mathrm{~d}, J=5.9 \mathrm{~Hz}, 1 \mathrm{H}, \mathrm{H}-3), 7.56(\mathrm{~d}, J=5.8 \mathrm{~Hz}$, $1 \mathrm{H}, \mathrm{H}-4), 7.18$ (s, 1H, H-6), $3.82\left(\mathrm{~s}, 3 \mathrm{H}, \mathrm{OCH}_{3}\right), 2.46$ (d, $\left.J=0.6 \mathrm{~Hz}, 3 \mathrm{H}, \mathrm{CH}_{3}\right) \mathrm{ppm} ;{ }^{13} \mathrm{C}$ NMR $(75.5 \mathrm{MHz}$, methanol$\left.d_{4}\right): \delta=147.7(\mathrm{C}-7), 147.1(\mathrm{C}-8), 144.4(\mathrm{C}-7), 141.6(\mathrm{C}-3)$, 139.8 (C-6), 135.0 (C-4a), 121.6 (C-8a), 121.2 (C-4), $118.9(\mathrm{C}-5), 61.0\left(\mathrm{OCH}_{3}\right), 17.2\left(\mathrm{CH}_{3}\right) \mathrm{ppm}$; HR-ESI-MS: calcd. $\mathrm{m} / \mathrm{z}$ for $\left[\mathrm{C}_{11} \mathrm{H}_{11} \mathrm{NO}_{2}+\mathrm{H}\right]^{+} 190.0863$, found 190.0862 .

\section{8-Hydroxy-7-methoxy-2,6-dimethylisoquinolinium} iodide (ETM-204, $\mathrm{C}_{\mathbf{1 2}} \mathrm{H}_{\mathbf{1 4}} \mathrm{INO}_{\mathbf{2}}$ ) A $25 \mathrm{~cm}^{3}$ round-bottom flask was charged with a solution of $300 \mathrm{mg}$ 7-methoxy6-methylisoquinolin-8-ol (11, $1.59 \mathrm{mmol}, 1.0 \mathrm{eq})$ in $7.9 \mathrm{~cm}^{3}$ abs. $\mathrm{MeOH}$. Iodomethane $\left(987 \mathrm{~mm}^{3}, 15.9 \mathrm{mmol}, 10 \mathrm{eq}\right)$ were added to the stirred orange solution (partially undissolved) and the tightly closed flask was stirred at $23{ }^{\circ} \mathrm{C}$ for $27 \mathrm{~h}$. The resulting orange solution was concentrated via a Schlenk line by condensating the volatiles in an intermediate cooling trap and the remaining yellow solid was dried in oil-pump vacuum to give $523 \mathrm{mg}$ ( $1.58 \mathrm{mmol}$, quant.) of the final compound ETM-204, which did not require further purification. $R_{f}=0.30$ (strong tailing; $\mathrm{CH}_{2} \mathrm{Cl}_{2} / \mathrm{MeOH}=10: 1$ (v/v); UV/CAM); m.p.: 201-203 ${ }^{\circ} \mathrm{C}$ (decomposition); ${ }^{1} \mathrm{H}$ NMR $\left(300 \mathrm{MHz}\right.$, methanol- $\left.d_{4}\right): \delta=9.73(\mathrm{~s}, 3 \mathrm{H}, \mathrm{H}-1), 8.32$ (dd, $J=6.8,1.2 \mathrm{~Hz}, 1 \mathrm{H}, \mathrm{H}-3), 8.16(\mathrm{~d}, J=6.8 \mathrm{~Hz}, 1 \mathrm{H}, \mathrm{H}-4)$, 7.56 (s, $1 \mathrm{H}, \mathrm{H}-5), 4.46$ (s, $\left.3 \mathrm{H}, \mathrm{N}-\mathrm{CH}_{3}\right), 3.88\left(\mathrm{~s}, 3 \mathrm{H}, \mathrm{OCH}_{3}\right)$, $2.60\left(\mathrm{~s}, 3 \mathrm{H}, \mathrm{CH}_{3}\right) \mathrm{ppm} ;{ }^{13} \mathrm{C}$ NMR $(75.5 \mathrm{MHz}$, methanol$\left.d_{4}\right): \delta=149.7(\mathrm{C}-8), 148.0(\mathrm{C}-7), 146.9(\mathrm{C}-6), 146.6(\mathrm{C}-1)$, 135.1 (2C, C-3 and C-4a), 125.5 (C-4), $120.9(\mathrm{C}-8 \mathrm{a}), 119.9$ $(\mathrm{C}-5), 61.3\left(\mathrm{OCH}_{3}\right), 48.3\left(\mathrm{~N}-\mathrm{CH}_{3}\right), 17.8\left(\mathrm{CH}_{3}\right) \mathrm{ppm}$; HRESI-MS: calcd. $m / z$ for $\left[\mathrm{C}_{12} \mathrm{H}_{14} \mathrm{NO}_{2}\right]^{+} 204.1019$, found 204.1019.

Acknowledgements We thank Ing. Carina Illaszewicz-Trattner and Prof. Dr. Jörg Weber for assistance in NMR measurements and NAWI Graz for financial support.

Funding Open access funding provided by Graz University of Technology.

Open Access This article is licensed under a Creative Commons Attribution 4.0 International License, which permits use, sharing, 
adaptation, distribution and reproduction in any medium or format, as long as you give appropriate credit to the original author(s) and the source, provide a link to the Creative Commons licence, and indicate if changes were made. The images or other third party material in this article are included in the article's Creative Commons licence, unless indicated otherwise in a credit line to the material. If material is not included in the article's Creative Commons licence and your intended use is not permitted by statutory regulation or exceeds the permitted use, you will need to obtain permission directly from the copyright holder. To view a copy of this licence, visit http://creativecommons. org/licenses/by/4.0/.

\section{References}

1. Corey EJ, Gin DY, Kania RS (1996) J Am Chem Soc 118:9202

2. Endo A, Yanagisawa A, Ab M, Tohma S, Kan T, Fukuyama T (2002) J Am Chem Soc 124:6552

3. Kawagishi F, Toma T, Inui T, Yokoshima S, Fukuyama T (2013) J Am Chem Soc 135:13684

4. Zheng S, Chan C, Furuuchi T, Wright BJD, Zhou B, Guo J, Danishefsky SJ (2006) Angew Chem Int Ed 45:1754

5. Zheng S, Chan C, Furuuchi T, Wright BJD, Zhou B, Guo J, Danishefsky SJ (2006) Angew Chem 118:1786

6. Chen J, Chen X, Bois-Choussy M, Zhu J (2005) J Am Chem Soc 128:87

7. Chen J, Chen X, Willot M, Zhu J (2006) Angew Chem Int Ed 45:8028

8. Jia J, Chen R, Jia Y, Gu H, Zhou Q, Chen X (2019) J Org Chem $84: 13696$

9. Fishlock D, Williams RM (2008) J Org Chem 73:9594

10. He W, Zhang Z, Ma D (2019) Angew Chem Int Ed 58:3972

11. Avendano C, de la Cuesta E (2010) Chem Eur J 16:9722

12. Cuevas C, Perez M, Martin M, Chicharro J, Fernandez-Rivas C, Flores M, Francesch A, Gallego P, Zarzuelo M, De La Calle F,
Garcia J, Polanco C, Rodriguez I, Manzanares I (2000) Org Lett $2: 2545$

13. Menchaca R, Martínez V, Rodríguez A, Rodríguez N, Flores M, Gallego P, Manzanares I, Cuevas C (2003) J Org Chem 68:8859

14. Reid JM, Kuffel MJ, Ruben SL, Morales JJ, Rinehart KL, Squillace DP, Ames MM (2002) Clin Cancer Res 8:2952

15. Brandon EFA, Sparidans RW, Guijt KJ, Löwenthal S, Meijerman I, Beijnen JH, Schellens JHM (2006) Investigat New Drugs 24:3

16. Beumer JH, Rademaker-Lakhai JM, Rosing H, Hillebrand MJX, Bosch TM, Lopez-Lazaro L, Schellens JHM, Beijnen JH (2007) Cancer Chemother Pharmacol 59:825

17. Vermeir M, Hemeryck A, Cuyckens F, Francesch A, Bockx M, Van Houdt J, Steemans K, Mannens G, Aviles P, De Coster R (2009) Biochem Pharmacol 77:1642

18. Cohen T, Dietz AG Jr, Miser JR (1977) J Org Chem 42:2053

19. Xiao Y, Xu Y, Cheon H-S, Chae J (2013) J Org Chem 78:5804

20. Werlé C, Yin C-JM, Heinemann FW, Hauser C, Meyer K (2017) Tetrahedron Lett 58:2715

21. Larrow JF, Jacobsen EN (2004) Org Synth Coll 10:96

22. Duff JC, Bills EJ (1934) J Chem Soc 1305. https://pubs.rsc.org/ en/content/articlelanding/1932/JR/jr9320001987

23. Hofsløkken NU, Skattebøl L (1999) Acta Chem Scand 53:258

24. Wang Y-X, Wang L, Xu Y-N, Li Y-H, Jiang J-D, Si S-Y, Li Y-B, Ren G, Shan Y-Q, Hong B, Song D-Q (2011) Eur J Med Chem 46:1066

25. Pomeranz C (1893) Monatsh Chem 14:116

26. Woodward RB, Doering WE (1945) J Am Chem Soc 67:860

27. Birch AJ, Jackson AH, Shannon PVR (1974) J Chem Soc. Perkin Trans 1:2185

Publisher's Note Springer Nature remains neutral with regard to jurisdictional claims in published maps and institutional affiliations. 